ARTICLE

DOI: $10.1038 / \mathrm{s} 41467-017-01247-3$

\title{
Structural and electronic determinants of lytic polysaccharide monooxygenase reactivity on polysaccharide substrates
}

\author{
T.J. Simmons ${ }^{1}$, K.E.H. Frandsen (10 9 ${ }^{9}$, L. Ciano (1) ${ }^{3}$, T. Tryfona ${ }^{1}$, N. Lenfant ${ }^{4,5}$, J.C. Poulsen ${ }^{2}$, L.F.L. Wilson (1) ${ }^{1}$, \\ T. Tandrup ${ }^{2}$, M. Tovborg ${ }^{6}$, K. Schnorr ${ }^{6}$, K.S. Johansen ${ }^{7}$, B. Henrissat ${ }^{4,5,8}$, P.H. Walton (10 ${ }^{3}$, \\ L Lo Leggio (i) $^{2} \&$ P. Dupree (i) ${ }^{1}$
}

Lytic polysaccharide monooxygenases (LPMOs) are industrially important copper-dependent enzymes that oxidatively cleave polysaccharides. Here we present a functional and structural characterization of two closely related AA9-family LPMOs from Lentinus similis (LSAA9A) and Collariella virescens (CVAA9A). LSAA9A and CVAA9A cleave a range of polysaccharides, including cellulose, xyloglucan, mixed-linkage glucan and glucomannan. LSAA9A additionally cleaves isolated xylan substrates. The structures of CVAA9A and of LSAA9A bound to cellulosic and non-cellulosic oligosaccharides provide insight into the molecular determinants of their specificity. Spectroscopic measurements reveal differences in copper co-ordination upon the binding of xylan and glucans. LSAA9A activity is less sensitive to the reducing agent potential when cleaving xylan, suggesting that distinct catalytic mechanisms exist for xylan and glucan cleavage. Overall, these data show that AA9 LPMOs can display different apparent substrate specificities dependent upon both productive protein-carbohydrate interactions across a binding surface and also electronic considerations at the copper active site.

\footnotetext{
${ }^{1}$ Department of Biochemistry, University of Cambridge, Cambridge, CB2 1QW, UK. ${ }^{2}$ Department of Chemistry, University of Copenhagen, Copenhagen, DK2100, Denmark. ${ }^{3}$ Department of Chemistry, University of York, York, YO10 5DD, UK. ${ }^{4}$ Architecture et Fonction des Macromolécules Biologiques (AFMB), CNRS, Aix-Marseille Université, Marseille, 13288, France. ${ }^{5}$ Institut National de la Recherche Agronomique (INRA), AFMB, Marseille, 13288, France. ${ }^{6}$ Research and Development, Novozymes A/S, Bagsvaerd, DK-2880, Denmark. ${ }^{7}$ Department of Geoscience and Natural Resources Management, Copenhagen University, Frederiksberg, DK-1958, Denmark. ${ }^{8}$ Department of Biological Sciences, King Abdulaziz University, Jeddah, 21589 , Saudi Arabia.

${ }^{9}$ INRA, UMR 1163 BBF (Biodiversité et Biotechnologie Fongiques), Marseille, 13288, France. Simmons T.J. and Frandsen K.E.H. contributed equally to this work. Correspondence and requests for materials should be addressed to L.L.L. (email: leila@chem.ku.dk) or to P.D. (email: pd101@cam.ac.uk)
} 
T he need for sustainable sources of energy and materials has spurred significant research efforts towards a greater understanding of the biological catabolism of lignocellulose, the world's most abundant source of renewable material and bioenergy ${ }^{1,2}$. The inherent recalcitrance of lignocellulose, however, is one of the major barriers to the utilization of biomass. This recalcitrance is a consequence of both the heterogeneous composition and the often semi-crystalline association of the polymers ${ }^{3,4}$. In addressing the problem of recalcitrance, multiple potential means have been proposed and assessed, including chemical, mechanical and enzymatic methods. Advances in enzyme cocktail formulations that accelerate the saccharification step of cell wall breakdown, in particular the inclusion of the lytic polysaccharide mono-oxygenases (LPMOs) ${ }^{6}$, are helping cellulosic-ethanol biorefineries move towards both commercial and environmental viability.

LPMOs are reducing agent- and $\mathrm{O}_{2}$-dependent copper metalloenzymes now classified as auxiliary activity families AA9-AA11 and AA1 $3^{7-11}$. Extensive spectroscopic and structural studies on LPMOs have shown that the enzyme's active site contains a single copper ion, which is coordinated by the amino terminus nitrogen atom, by a side chain nitrogen atom of the $\mathrm{N}$-terminal histidine, and by the side chain nitrogen atom of an additional histidine, in a structural motif known as the histidine brace ${ }^{12}$. What is distinctive about LPMOs is that they oxidatively rather than hydrolytically cleave polysaccharides producing saccharides with oxidized ends ${ }^{13}$. LPMOs augment the action of other polysaccharide-degrading enzymes, and accordingly much research attention is devoted to a greater understanding of the enzymatic mechanism and the range of LPMO saccharide substrates.

It was first shown that LPMOs could boost the action of cellulases on cellulose and chitin ${ }^{14-16}$, but LPMOs are now known to act on several crystalline substrates such as chitin, cellulose and retrograded starch ${ }^{10-12,17}$. Later, enzymes with activity against non-crystalline and oligomeric structures were identified ${ }^{8}, 19$. Furthermore, fungal AA9 LPMOs have been shown to be active on soluble substrates such as xyloglucan, mixed-linkage glucan and glucomannan ${ }^{19-22}$, and on cellulose-bound xylan ${ }^{23}$. Conspicuously, an LPMO active on isolated xylan has not been reported; however, this range of reported substrates will likely grow. The large number and sequence diversity of LPMOs that individual fungi maintain ${ }^{24}$, and their disparate expression profiles when the fungi are grown on different polysaccharide substrates $^{25}$, 26, signal that AA9 LPMOs do have distinct, and functionally significant, polysaccharide substrate specificities, although some evolutionary diversity of LPMOs likely arises through their use of different reducing systems $\mathrm{s}^{27}, 28$.
The root causes of LPMO substrate specificity remain poorly understood. This is because LPMO chemistry is a subtle and complex combination of structural and electronic factors, both of which must be taken into account when developing an understanding of the mechanism of action ${ }^{29}$. The structure-function relationship of substrate specificity and regiospecificity has been recently reviewed ${ }^{30,} 31$. Insight into LPMO:substrate binding can be gained from the structures of LPMOs ${ }^{30}$ and combined structural and spectroscopic studies of LPMOs in contact with substrate. Recent ITC, NMR and docking studies of an AA9 LPMO from Neurospora crassa in contact with oligosaccharides revealed that more extended substrates had significantly higher binding affinities. This is in accord with a multi-point interaction of the substrate with the LPMO surface where the surface loops in some LPMOs remote from the active site enhance binding affinity ${ }^{21}$ The study also showed that a single cellohexaose $\left(\right.$ Cell $\left._{6}\right)$ chain likely spans the copper active site from the -3 to +3 or -2 to +4 subsites (subdivisions of the binding cleft numbered relative to the site of cleavage ${ }^{32}$ ), in which the L3 loop (important for interactions with the $+3 /+4$ subsites) and the LC loop (important for binding to approximately -4 subsite) lie at somewhat extended distances from the copper active site. Detailed insight into an AA9 LPMO-substrate interaction came from the first crystal structures of LPMO:oligosaccharide complexes: Lentinus similis AA9A (LsAA9A) bound to $\mathrm{Cell}_{6}$ and $\mathrm{Cell}_{3}{ }^{33}$. Cell 6 was shown to bind at subsites -4 to +2 via interactions with aromatic residues, the N-terminal His and a conserved Tyr as well as a number of hydrogen-bonds with other residues in a contoured binding surface on the LPMO. The +2 glucosyl residue exhibits a set of well-defined hydrogen-bonding interactions with amino-acid side chains (Asn28, His66 and Asn67) essentially locking this residue into a fixed position with respect to the active site.

Electronic factors around the active site also play a key role in determining the reactive mechanism. Changes in the electronic structure of the copper ion, an important factor in the ability of the copper ion to activate $\mathrm{O}_{2}$, occur upon substrate binding to LsAA9A ${ }^{33}$. Furthermore, in an illustration of the complexity of substrate-LPMO interaction and the subtle interplay of electronic and structural factors, Cell $_{6}$ is bound synergistically with an exogenous ligand on the copper ion. It is likely that the oxidative mechanism adopted by LPMOs can proceed via one or more of several different routes ${ }^{34}$, the determinants of which depend on the varying extents of the substrate, the reducing agent reducing the potential and the positioning of the substrate on the LPMO surface. For instance, the means by which electrons are donated to the LPMO active site modulate the apparent range of reactivity ${ }^{28,35}$.

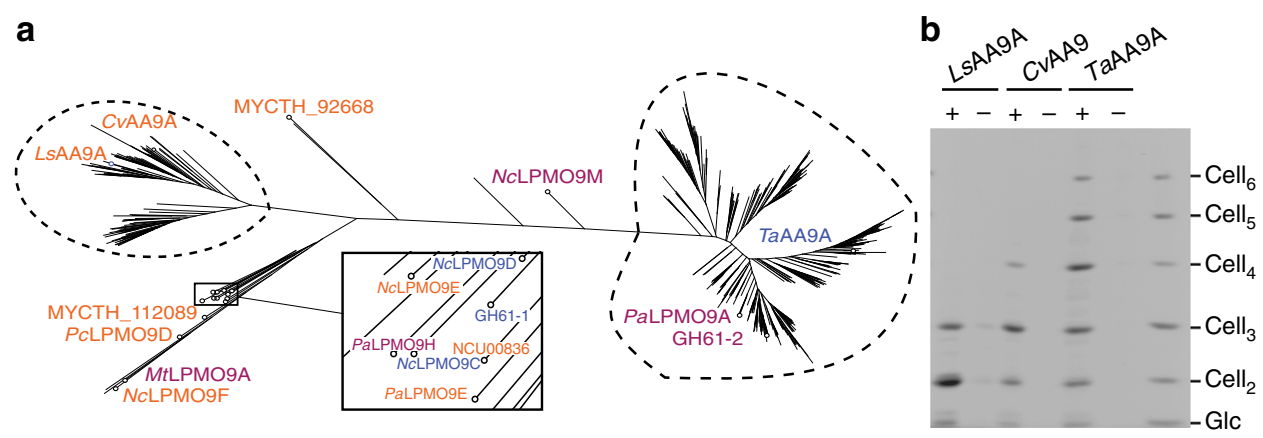

Fig. 1 Sequence similarity between $\mathrm{LSAA9A}$, CVAA9A and TaAA9A, and analysis of their reaction products. a Distance tree of 444 selected AA9 sequences (see Methods). Blue, purple and orange labels designate $A A 9$ enzymes that oxidize the sugar ring at $C 1, C 4$ and $C 1+C 4$, respectively. See Supplementary Table 1 for protein accession numbers. Unlabelled branches represent AA9 enzymes for which the regioselectivity of oxidation is not available from the literature. b PACE gel showing reaction products of the three enzymes on PASC; +, incubation with 4 mM ascorbate; -, incubation without ascorbate (performed in triplicate) 

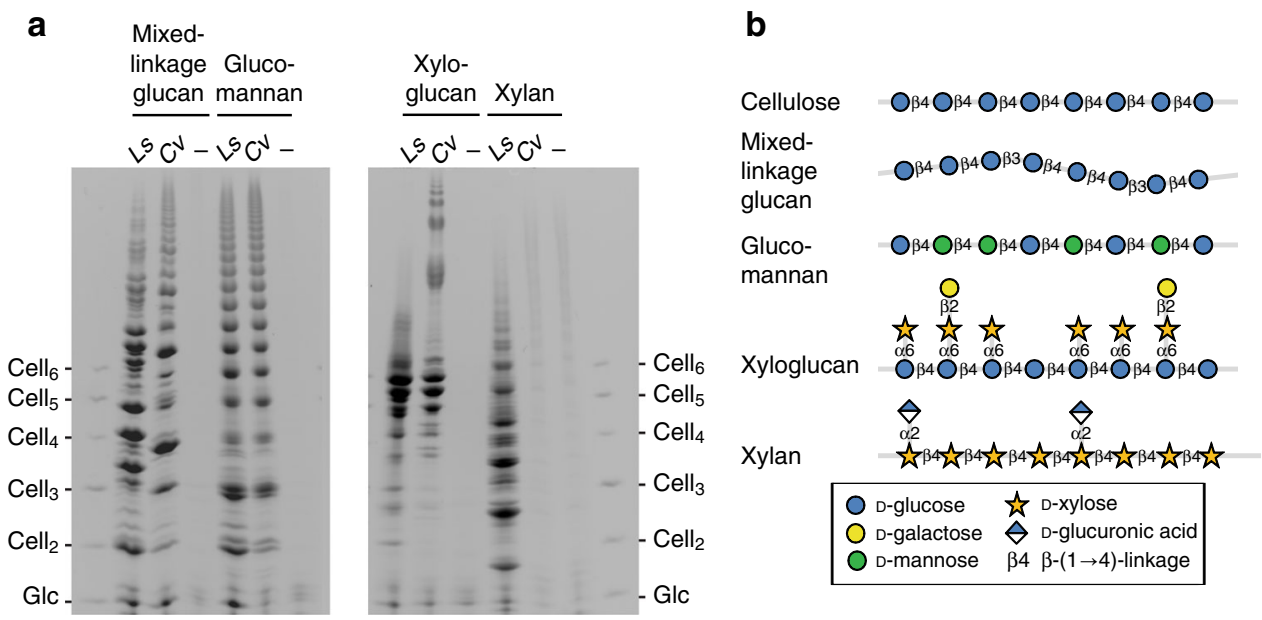

Fig. 2 Comparison of LSAA9A and CVAA9A action on non-cellulosic substrates. a PACE gel showing digestion products on lignocellulosic polysaccharides with $4 \mathrm{mM}$ ascorbate reducing agent. Ls, LsAA9A; CV, CVAA9A; -, no enzyme (performed in triplicate). The migration standards are cello-oligosaccharides. b Structures of polysaccharides

The detailed molecular and electronic insights of the LPMO-substrate interaction afforded by combined biochemical, $\mathrm{X}$-ray diffraction and electron paramagnetic resonance (EPR) spectroscopic studies can significantly enhance our understanding of LPMO reactivity. We report herein a study into the principal structural and electronic factors of the reactivity of two AA9 LPMOs with a range of substrates. Through X-ray crystal structures studies of LsAA9A with bound substrates, we illustrate how binding cleft interactions dictate the site of polysaccharide attack. Through comparison with the $C v \mathrm{AA} 9 \mathrm{~A}$ structure, also determined here, we suggest some structural determinants of specificity for the two enzymes. LsAA9A is active on isolated xylan, but this activity is associated with a distinct low sensitivity to reducing agent potential and a different copper co-ordination at the active site, which together reveal an alternative mechanistic pathway for LPMO action on this substrate. These data show how AA9 LPMO substrate cleavage is dependent upon both productive protein:carbohydrate interactions across a binding surface and also electronic considerations at the active site.

\section{Results}

CvAA9A is an AA9 LPMO active on cello-oligosaccharides. To help understand the basis of AA9 substrate specificity, we searched for enzymes related to LsAA9A that might also cleave soluble oligosaccharides. Because LPMOs exhibit high variability in their C-termini, we performed a large-scale alignment of LPMO protein sequences using their $\mathrm{N}$-terminal portion ${ }^{36}$. We selected 98 AA9 sequences that were highly similar in their $\mathrm{N}$ terminal half to LsAA9A and 326 sequences that were highly similar to TaAA9A in the same region. TaAA9A was used for comparison purposes, as it does not show the ability to cleave soluble oligosaccharides. After adding the sequences of 20 AA9 enzymes studied in the literature, a distance tree was built with the resulting 444 AA9 sequences (Fig. 1a, Supplementary Table 1 ). The tree clearly places $L s A A 9 A$ and TaAA9A in distinct clades. From the LsAA9A clade, we identified an LPMO from Collariella virescens (CvAA9A; $46 \%$ sequence similarity to LsAA9A) that lacks some residues observed by Frandsen et al. ${ }^{33}$ as being involved in enzyme-substrate interactions (Supplementary Fig. 1). All three of the subsite +2 substrate-binding residues in LsAA9A (Asn28, His66 and Asn67) are different in CvAA9A (Thr28, Arg67 and Val68) (Supplementary Fig. 1). To study the activity of CvAA9A, the enzyme was expressed in Aspergillus oryzae and successfully purified from the fermentation broth.
(Note that the expression in this fungal host preserves the natural side-chain methylation at the $\mathrm{N}$-terminal histidine, in contrast to fungal LPMO expression in Pichia pastoris and bacterial systems.) On phosphoric acid-swollen cellulose (PASC), CvAA9A produced a range of cello-oligosaccharides (Fig. 1b). The cellooligosaccharide product profile of $C v \mathrm{AA} 9 \mathrm{~A}$ was similar to that of LsAA9A and notably shorter than those produced by TaAA9A. Indeed, $\mathrm{CvAA9A}$ readily degraded $\mathrm{Cell}_{6}$-(2-aminobenzamide) $\left(\mathrm{Cell}_{6}-2 \mathrm{AB}\right)$ using a C4-oxidising mechanism to yield $\mathrm{Cell}_{3}$ and oxidized $\mathrm{Cell}_{3}-2 \mathrm{AB}$ (Supplementary Fig. 2), like LsAA9A but unlike TaAA9A ${ }^{33}$. Therefore, the distance relationships between the three enzymes, as measured using the $\mathrm{N}$-terminal comparison method above, mirror the similarities in activities of the enzymes.

Position-specific cleavage of a range of hemicelluloses. We next determined whether the $L s \mathrm{AA} 9 \mathrm{~A}$ and $C v \mathrm{AA} 9 \mathrm{~A}$ enzymes are active on a range of $\beta$ - $(1 \rightarrow 4)$-D-glucan-related polysaccharides (Fig. 2, Table 1). Mixed-linkage glucan (MLG) is a $\beta$-D-glucan in which three to four $(1 \rightarrow 4)$-linked residues $\left(\mathrm{Cell}_{3}, \mathrm{Cell}_{4}\right)$ are separated by single $(1 \rightarrow 3)$ bonds, glucomannan has a backbone randomly composed of $\beta$-( $(1 \rightarrow 4)$-D-glucosyl and $\beta$ - $(1 \rightarrow 4)$-Dmannosyl residues, xyloglucan is a $\beta-(1 \rightarrow 4)$-D-glucan with $\alpha$-(1 $\rightarrow 6)$-D-xylosyl branches, and xylan is a polymer of $\beta-(1 \rightarrow$ 4)-D-xylosyl residues that is similar to $\beta$-( $1 \rightarrow 4)$-D-glucan but lacks C6 groups (Fig. 2b). Both LsAA9A and CvAA9A showed activity against MLG, glucomannan and xyloglucan, producing a range of oligosaccharide products (Fig. 2a). LsAA9A also showed some activity on xylan, whereas $C v$ AA9A showed no measurable activity on this substrate. No LPMO activity was observed on starch $(\alpha-(1 \rightarrow 4)$-D-glucan $)$, laminarin $(\beta$ - $(1 \rightarrow 3)$-D-glucan $)$ or chitin (poly $\beta-(1 \rightarrow 4)$-D-GlcNAc) (Supplementary Fig. 3). Altogether, these activities indicate that both $L s \mathrm{AA} 9 \mathrm{~A}$ and $\mathrm{CvAA9A}$ enzymes only cleave near $\beta$ - $(1 \rightarrow 4)$ bonds, and that some variation to the cellulosic $\beta$-(1 $\rightarrow 4)$-D-glucan, including substitution, linkage and backbone residue, can be accommodated at or near the site of cleavage by both of the enzymes.

To identify precise substrate cleavage sites, we studied the products of both LsAA9A and CvAA9A cleavage of MLG, glucomannan, xyloglucan and xylan (in the case of LsAA9A) polysaccharides by MALDI-ToF MS. Minor double oxidation products were observed, indicating cleavage of these hemicelluloses and PASC using both $\mathrm{C} 1$ - and C4-oxidising mechanisms (Fig. 3; Supplementary Fig. 4, Table 1). We further investigated the site of attack on these different hemicelluloses using differing 


\section{Table 1 Summary of activity assays on different substrates}

\begin{tabular}{|c|c|c|c|c|}
\hline \multirow[t]{2}{*}{ Polysaccharide } & \multicolumn{2}{|l|}{ LsAA9A } & \multicolumn{2}{|l|}{ CvAA9A } \\
\hline & Activity & Notes & Activity & Notes \\
\hline Cellulose and cello-oligosaccharides & ++ & $\begin{array}{l}\text { Activity on both cellulose oligosaccharides and insoluble } \\
\text { cellulose material (PASC) }\end{array}$ & ++ & $\begin{array}{l}\text { Activity on both cellulose oligosaccharides and insoluble } \\
\text { cellulose material (PASC) }\end{array}$ \\
\hline MLG & ++ & $\begin{array}{l}\text { Pattern suggests } \beta \text { - }(1 \rightarrow 3) \text { bonds accommodated at specific } \\
\text { places within active site, but not between }-1 \text { and }+1\end{array}$ & ++ & $\begin{array}{l}\text { Pattern suggests } \beta-(1 \rightarrow 3) \text { bonds accommodated at specific } \\
\text { places within active site, but not between }-1 \text { and }+1\end{array}$ \\
\hline Glucomannan and $\mathrm{Man}_{6}$ & ++ & $\begin{array}{l}\text { Cleavage can occur with Glc or mannose at }-1 \text { or }+1 \text {. Inactivity } \\
\text { on Man }{ }_{6} \text { indicates some Glc C } 2 \text { hydroxyl orientation needs to } \\
\text { be present between }-3 \text { and }+3\end{array}$ & ++ & 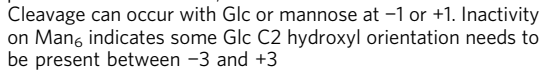 \\
\hline Xyloglucan & ++ & $\begin{array}{l}\text { Cleavage occurs with unsubstituted Glc at subsite }+1 \text {. Xylosyl } \\
\text { substitution at }-3,-2,-1,+2 \text { and }+3 \text {. Galactosyl-xylosyl } \\
\text { substitutions can occur at either }-2,-1 \text { and/or }+3\end{array}$ & ++ & $\begin{array}{l}\text { Cleavage occurs with unsubstituted Glc at subsite }+1 \text {. Xylosyl } \\
\text { substitution at }-3,-2,-1,+2 \text { and }+3 \text {. Galactosyl-xylosyl } \\
\text { substitutions can occur at either }-2,-1 \text { and/or }+3\end{array}$ \\
\hline Xylan and $\mathrm{Xyl}_{6}$ & + & $\begin{array}{l}\text { Activity on both. Weak } \mathrm{Xyl}_{6} \text { activity compared to } \mathrm{Cell}_{6} \\
\text { suggests that, while Glc } \mathrm{C} 6 \text { is not required for activity, it is } \\
\text { very important at certain sites, such as }+1\end{array}$ & $+/-$ & Much poorer activity of CVAA9A compared with LsAA9A \\
\hline Starch & - & $\begin{array}{l}\text { Absence of activity indicates that LsAA9A necessarily cleaves } \\
\beta-(1 \rightarrow 4) \text { bonds }\end{array}$ & - & $\begin{array}{l}\text { Absence of activity indicates that CVAA9A necessarily cleaves } \\
\beta-(1 \rightarrow 4) \text { bonds }\end{array}$ \\
\hline Laminarin & - & & - & \\
\hline $\begin{array}{l}\text { G4G3G4G (MLG oligosaccharide) } \\
\text { Chitin }\end{array}$ & - & & - & \\
\hline Chitin & & $\begin{array}{l}\text { Absence of activity indicates that } L S A A 9 A \text { either requires } \mathrm{O} 2 \\
\text { interactions or cannot accommodate } \mathrm{N} \text {-acetyl on amino } \mathrm{C} 2\end{array}$ & - & $\begin{array}{l}\text { Absence of activity indicates that } \mathrm{CrAA9A} \text { either requires } \mathrm{O} 2 \\
\text { interactions or cannot accommodate } \mathrm{N} \text {-acetyl on amino } \mathrm{C} 2\end{array}$ \\
\hline
\end{tabular}

a

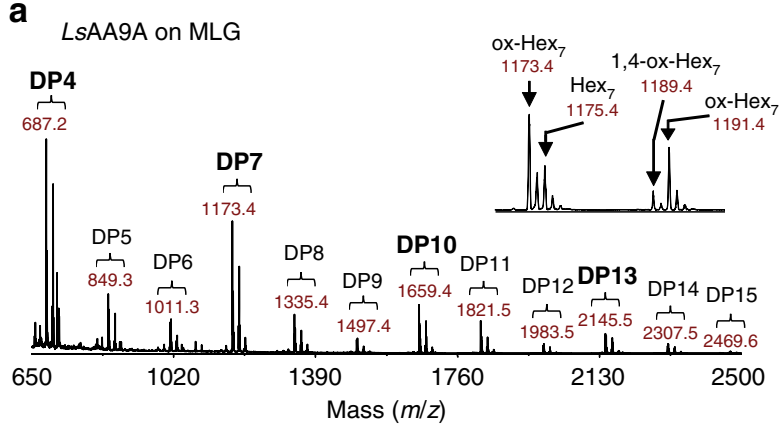

b CVAA9A On MLG

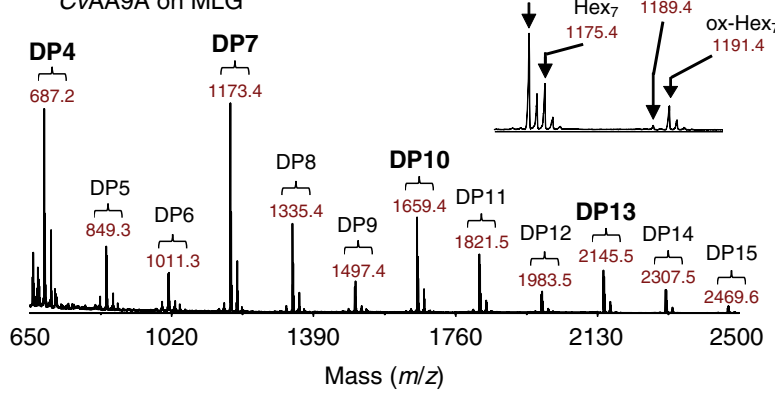

c

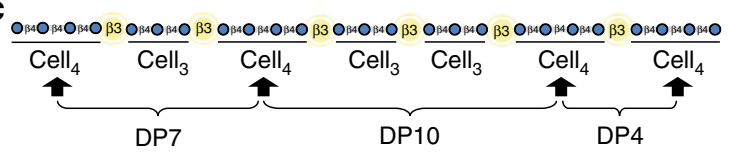

Fig. 3 LsAA9A and CvAA9A digestion products of MLG suggest preference for Cell $_{4}$ region cleavage. Products of LSAA9A (a) and CVAA9A (b) activity on barley MLG with $4 \mathrm{mM}$ ascorbate were analysed by MALDI-ToF MS (performed in triplicate). Both enzymes can produce both $\mathrm{C} 1$ and C4 oxidation on MLG (1,4-ox; oxidized C1 and C4, see insets). Further, oligosaccharide profiles show a distinct pattern indicative of the mechanism of attack and substrate specificity of each enzyme on MLG. c Proposed region of cleavage

protocols. On MLG, we observed in the MALDI data a predominance of DP 4, 7 and 10 oligosaccharides indicating that each enzyme favours cleaving within $\mathrm{Cell}_{4}$ regions over $\mathrm{Cell}_{3}$ regions (Fig. 3). The inability of $L s A A 9 A$ and $C v A A 9 A$ to cleave $\beta$-Glc- $(1 \rightarrow 4)$ - $\beta$-Glc- $(1 \rightarrow 3)-\beta$-Glc- $(1 \rightarrow 4)$-Glc

(G4G3G4G), despite their ability to cleave Cell 4 (G4G4G4G) (Supplementary
Fig. 5), supports the hypothesis that neither enzyme can cleave at $\beta$ - $(1 \rightarrow 3)$ bonds and require substantial $\beta$ - $(1 \rightarrow 4)$-linked regions for cleavage. On glucomannan, we employed High-Performance Anion-Exchange Chromatography (HPAEC) analysis of trifluoroacetic acid (TFA) hydrolysates of digestion products to assess the site of cleavage. Notably, the data indicated that cleavage can occur not only between glucosyl residues, but also with mannose at the +1 or -1 subsite (Supplementary Fig. 6). In order to deduce site of attack on xyloglucan we employed xyloglucan DP14-18 oligosaccharides (Supplementary Fig. 7). Inspection of the position of substituted glucose (Glc) in the products indicated that xylosyl substitution of Glc at O-6 was accommodated at the $-3,-2,-1,+2$ and +3 subsites but unsubstituted Glc was always required at subsite +1 . In contrast to the LsAA9A and CvAA9A products on polysaccharides, $L s \mathrm{AA} 9 \mathrm{~A}$ degraded $\mathrm{Xyl}_{6}-2 \mathrm{AB}$ to yield two trimers using solely a C4-oxidising mechanism (Supplementary Fig. 8), analogous to cleavage of Cell $_{6}-2 \mathrm{AB}$ by both LsAA9A ${ }^{33}$ and CvAA9A (Supplementary Fig. 2).

To allow a semi-quantitative determination of the influence of sugar structures on enzyme activity, we probed LsAA9A and CvAA9A cleavage of the soluble Cell ${ }_{6}$, xylohexaose $\left(\mathrm{Xyl}_{6}\right)$ and mannohexaose $\left(\mathrm{Man}_{6}\right)$ oligosaccharides (Supplementary Fig. 9). The LsAA9A activity against Cell 6 was substantially ( 100 -fold) better than its activity on $\mathrm{Xyl}_{6}$. Consistent with the absence of activity on glucuronoxylan, $\mathrm{C} v \mathrm{AA} 9 \mathrm{~A}$ activity on $\mathrm{Xyl}_{6}$ was almost undetectable ( $\sim 1000$-fold less than Cell ${ }_{6}$ activity). Although both enzymes showed activity on glucomannan and can cleave adjacent to mannose, activity was scarcely detectable on $\mathrm{Man}_{6}$ $\left(\sim 10,000\right.$-fold less than $\left.\mathrm{Cell}_{6}\right)$, indicating that the enzymes require some Glc residues within a mannan backbone for activity.

Recent results show dependence of the LPMO action on reductant strength ${ }^{28,37}$. We found that cleavages of MLG, glucomannan and xyloglucan by LsAA9A were sensitive to reducing agent potential, with ascorbate as reductant yielding much higher amount of product (Fig. 4a). In contrast, cleavage of xylan was not sensitive. We corroborated this finding with oligosaccharides, observing that $\mathrm{Xyl}_{6}$ was poorly sensitive to reducing agent potential, unlike cleavage of Cell 6 where $L s \mathrm{AA} 9 \mathrm{~A}$ showed much greater activity with ascorbate than pyrogallol ${ }^{33}$ (Fig. $4 \mathrm{~b})$.

LsAA9A: and CvAA9A:cello-oligosaccharide structures. To help understand the structural basis of LPMO attack on different substrates, we employed crystallographic analyses. We report here an LsAA9A:Cell ${ }_{5}$ complex (Fig. 5a, b; Supplementary Table 4; Table 2), which, owing to a lack of significant substrate contacts 
a

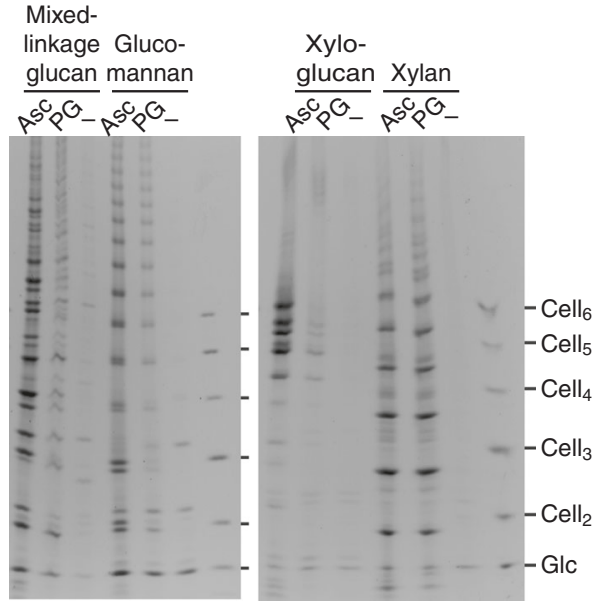

b

$$
\frac{\text { Asc }}{L s-} \frac{P G}{L s-} \frac{\text { Cys }}{L s-}
$$$$
\frac{\text { Asc }}{L s-} \frac{P G}{L s-} \frac{\text { Cys }}{L s-}
$$

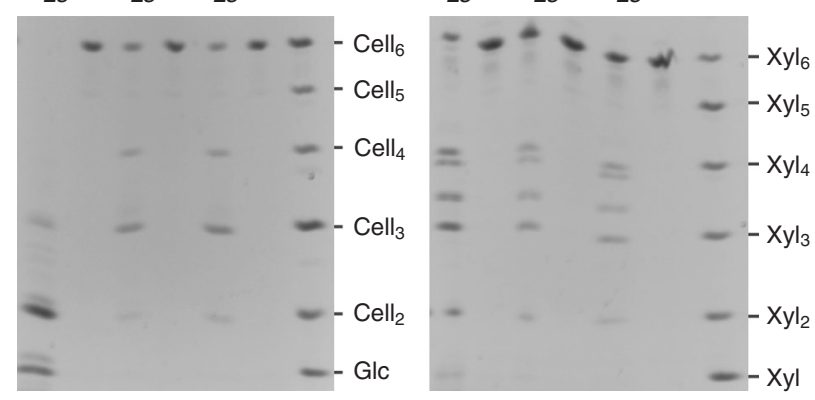

Fig. 4 LSAA9A activity on different poly- and oligosaccharide substrates show differing sensitivity to reducing agent potential. a PACE gels showing products of LSAA9A activity on MLG, glucomannan, xyloglucan and xylan polysaccharides using $4 \mathrm{mM}$ ascorbate or $4 \mathrm{mM}$ pyrogallol as reductants (performed in triplicate). The migration standards are cello-oligosaccharides. b PACE gels showing products of LsAA9A activity on Cell 6 and Xyl6 oligosaccharides using $4 \mathrm{mM}$ ascorbate, pyrogallol and cysteine as reductants (performed in triplicate). Asc Ascorbate; Cys cysteine; PG pyrogallol. The migration standards are cello- and xylo-oligosaccharides

to symmetry-related molecules, is a more faithful depiction of the binding conformation of a single oligosaccharide to LsAA9A as compared to the original LsAA9A:Cell 6 structure described by Frandsen et al. ${ }^{33}$. Tyr203 stacking is still a major interaction in LsAA9A:Cell 5 but a new hydrogen bond is seen between $\mathrm{O} 6$ and Asp150 at subsite -3 , and glycosidic torsion angles are closer to ideal values (Supplementary Table 2). Other interacting residues at the negative subsites are Glu148, Arg159 and Ser77 (Fig. 5; Supplementary Table 3). Like the LsAA9A:Cell 6 structure $^{33}$, the main interactions to $\mathrm{Cell}_{5}$ are a network of hydrogen bonds by Asn28, His66 and Asn67 interacting with $\mathrm{O} 2$ and $\mathrm{O} 3$ at subsite +2 , and the interaction with MeHis1 at subsite $+1^{38}$.

To understand better how protein structure might influence the similarities and differences in CvAA9A and LsAA9A substrate cleavage patterns, the X-ray crystal structure of CvAA9A was solved (Supplementary Figs. 10 and 11; Supplementary Tables 3 and 4; Table 2). The Cu-coordinating amino-acid residues are MeHis1 and His79 (with equatorial distances to the $\mathrm{Cu}$ ranging from 2.0 to $2.1 \AA$ ), while a non-coordinating Tyr169 occupies the axial position (2.6-2.8 $\AA$ ). No exogenous ligands are evident within $3.0 \AA$ of the $\mathrm{Cu}$-ion indicating that the active site is mostly in a photoreduced $\mathrm{Cu}(\mathrm{I})$ state. A 'pocket-water' is bound in an $\mathrm{H}$ bond network with the amide-nitrogen and oxygen of Asp76 and MeHis1, respectively. The active-site geometry of $C v$ AA9A thus closely resembles that of LsAA9A (Supplementary Fig. 10c). However, there are some amino-acid differences in CvAA9A compared to LsAA9A at subsites +2 and -1 . Crystals of CvAA9A were soaked with $\mathrm{Cell}_{3}$ and $\mathrm{Cell}_{6}$ oligosaccharides but this did not result in any catalytically relevant complex.

LsAA9A:hemicellulose oligosaccharide structures. To study the structural determinants of the LsAA9A positional specificity of cleavage, a number of LsAA9A crystal structures in complex with MLG, glucomannan and xylo-oligosaccharides were solved (see Table 2, Supplementary Fig. 12 and Supplementary Tables 2 and 4 for experimental and crystallographic data and refinement information, hydrogen-bonding interactions between enzyme and ligand, and ligand conformations). Soaking experiments with commercially available xyloglucan fragments failed to produce crystallographic complexes, possibly because the substrate oligosaccharides are large and binding likely to be impeded by crystal contacts.
Complexes with MLG tetrasaccharide. LsAA9A crystals were soaked with two different MLG tetrasaccharides, each with a single $\beta-(1 \rightarrow 3)$ linkage: G4G4G3G and G4G3G4G. Interestingly, the LsAA9A:G4G4G3G complex did not reveal any $\beta-(1 \rightarrow 3)$ linkages. An apparent $\mathrm{Cell}_{4}$ substrate appears to be bound from subsite -2 to +2 (Supplementary Fig. 13) giving essentially identical interactions as the -2 to +2 glucosyl residues in the LsAA9A:Cell ${ }_{5}$ complex. We interpret this result as the $\beta-(1 \rightarrow 4)$ glucan $\left(\mathrm{Cell}_{3}\right)$ part of the substrate being bound in two overlapping conformations in different asymmetric units from subsites -2 to +1 and -1 to +2 , while the $\beta$ - $(1 \rightarrow 3)$-glucosidic residues are completely disordered in both cases. A structure of LsAA9A crystals soaked with G4G3G4G could not be convincingly modelled, further indicating that the enzyme needs at least two consecutive $\beta$ - $(1 \rightarrow 4)$ linkages (a Cell 3 unit) for recognition and efficient binding.

Complexes with glucomannan oligosaccharides. LsAA9A crystals were soaked with a mixture of glucomannan oligosaccharides. The resulting difference density was well defined clearly showing glycosyl units occupying subsites -3 to +2 , additional density at -4 and some residual density occupying subsite +3 (Fig. $5 c$, e). Consistent with the activity data, the structure unequivocally showed a mannosyl unit at the +1 subsite, while glucosyl units were clearly observable at $-2,-1$ and +2 subsites. Moreover the $\mathrm{C} 2$ hydroxyl of the mannosyl unit at subsite +1 points towards the face of the imidazole side chain of MeHis1, and the axial water molecule is displaced (Fig. 5d, f). The identity of the glycosyl unit at subsite -3 is ambiguous though best modelled as mannose. The density of the glycosyl unit at subsite -4 is weak and occupies a very similar position as the corresponding unit in the LsAA9A:Cell ${ }_{6}$ complex, as does the glycosyl unit at subsite -3 , due to similar crystal constraints.

Complexes with xylo-oligosaccharide. Although in crystals soaked with $\mathrm{Xyl}_{3}$ and $\mathrm{Xyl}_{4}$ the oligosaccharides did not fully span the active site, $L s A A 9 A: \mathrm{Xyl}_{5}$ crystals revealed very welldefined density from subsites -3 to +2 (Fig. 6a). The oligosaccharide position at subsites -3 to -1 are similar to LsAA9A:Cell 5 , but with a translation of about half a pyranose unit in the non-reducing end direction. In contrast, the plane of the xyloside unit at subsite +1 is rotated $\sim 90^{\circ}$ compared to the corresponding glucosidic unit, while the xyloside residue binding +2 is rotated $\sim 180^{\circ}$ (Fig. 6d; Supplementary Table 2). As a result 


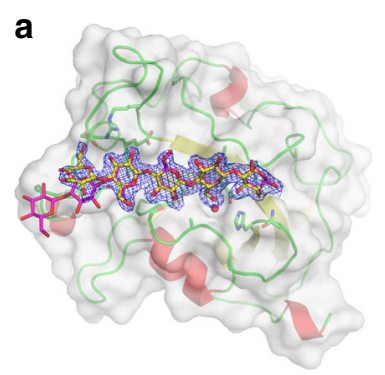

b
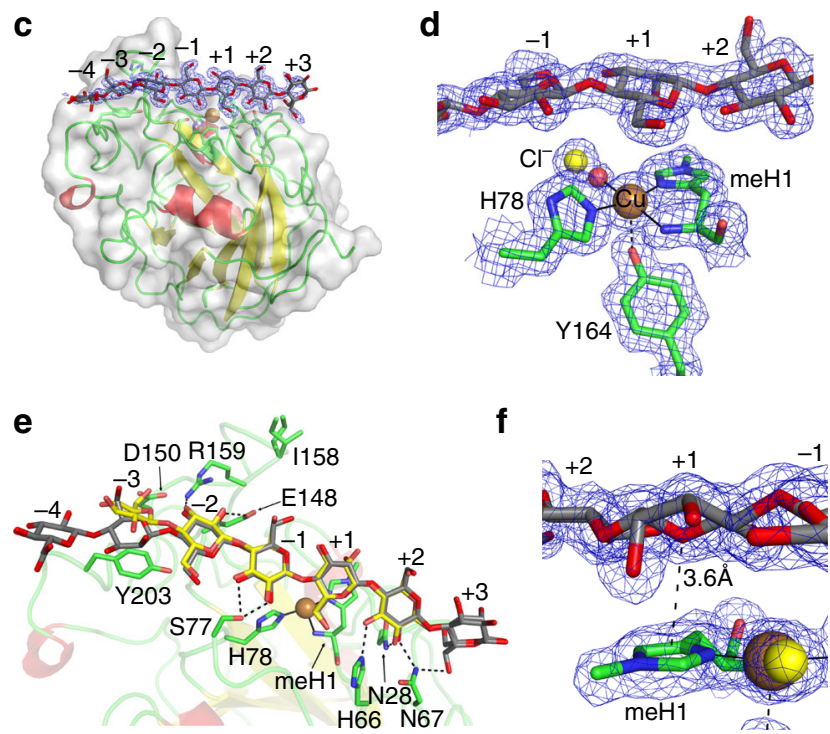

$\mathbf{f}$

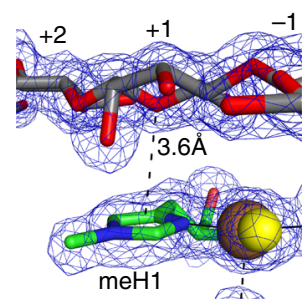

Fig. 5 Structures of $L s A A 9 A: C e l_{5}$ and $L s A A 9 A: g l u c o m a n n a n$ oligosaccharide complexes. a Cell 5 (yellow) is well defined in subsites -3 to +2. A $2 F_{\text {obs }}-F_{\text {calc }}$ electron density map is shown at $1 \sigma$ contour level. The structure shows no crystal contact induced distortion of the Cell ${ }_{5}$ substrate when compared to Cell $_{6}$ (magenta). b, LsAA9A-Cell 5 interactions are shown as dashes. An additional interaction between subsite -306 and Asp150 is gained in the absence of symmetry related contacts to the substrate. c Overall structure of LsAA9A with GM (grey) bound from subsite -4 to +3 . d Close-up (subsite -1 to +2 ) of the active site with GM fragment bound. Axial coordinations are in black dashes while equatorial coordinations are in full black lines. e Top-down view of LsAA9A:GM (GM in grey) and for comparison LSAA9A:Cell $_{5}$ (Cell ${ }_{5}$ in yellow). Dashed lines show interactions within hydrogen bond distance $(2.8 \AA)$. f The C2hydroxyl of mannose is clearly visible in the density at subsite +1 . The pyranose 05-imidazole ring interaction ( $3.6 \AA$ ) is indicated with dashes. The interaction of MeHis and the mannosyl residue is very similar to the interaction with glucosyl residues in previous complexes ${ }^{38}$. A $2 F_{\text {obs }}-F_{\text {calc }}$ electron density map is shown at $1 \sigma$ contour level for panels $\mathbf{a}, \mathbf{d}$ and $\mathbf{f}$

xylose at subsite +1 does not stack with MeHis1, and in fact appears to have no interactions with the enzyme, while the same residues that bind the subsite +2 glucosyl residue in the LsAA9A:

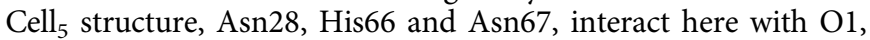
$\mathrm{O} 5$ and $\mathrm{O} 1$ of the +2 xyloside residue, respectively (Fig. 6c; Supplementary Table 3). A structure of $L s \mathrm{AA} 9 \mathrm{~A}: \mathrm{Xyl}_{5} \mathrm{Cu}$ (II) determined from a low X-ray dose data collection showed the substrate bound similarly, and revealed a mix of water $/ \mathrm{Cl}^{-}$in the axial position and a fully occupied equatorial water on the active site copper (Fig. 6b). Thus, in contrast to binding of celloor glucomannan oligosaccharides, the axial water was not displaced by binding of $\mathrm{Xyl}_{5}$.

EPR data suggest alternative $L s A A 9 A$ substrate binding modes. We studied substrate binding on both LsAA9A and CvAA9A using EPR spectroscopy (Table 3) to investigate the electronic state of the active site copper upon binding. As has been shown by Frandsen et al. ${ }^{33}$ and Courtade et al. ${ }^{21}$, the binding affinity of oligosaccharide substrates is significantly affected by the presence of the exogenous ligand on the copper ion. Accordingly, EPR experiments were carried out in both the absence and presence of $200 \mathrm{mM}$ chloride (1.0 M chloride for $\mathrm{Xyl}_{6}$ studies). Furthermore, experiments were carried out at high substrate concentration to maximize substrate binding. For LsAA9A a wide range of substrates was tested. In all cases, the parallel region of the spectra could be modelled with reliable $g_{z}$ and $\left|A_{z}\right|$ values, giving some insight into the electronic nature of the copper ion. Perpendicular values were less reliable due to the second-order nature of the spectra in this region, and are therefore not used in the analysis, although the appearance of superhyperfine coupling to ligands in this region was apparent in some cases (Table 3, Supplementary Fig. 14) and used as an indication of increased metal-ligand covalency in the singly-occupied molecular orbital (SOMO), as previously discussed by Frandsen et al. ${ }^{33}$. In all cases apart from xylan, the addition of substrate gave perturbation of the $\mathrm{Cu}$ spin Hamiltonian parameters similar to that already reported by Frandsen et al. ${ }^{33}$ In particular, shifts in $g_{z}$ values to ca 2.23 (along with the appearance of strong superhyperfine coupling) were seen upon addition of Avicel, glucomannan and xyloglucan, indicative of chloride coordination to the copper ion in the equatorial position of the copper coordination sphere. These shifts are analogous to those of $L s \mathrm{AA} 9 \mathrm{~A}$ interacting with $\mathrm{Cell}_{6}$ and $\mathrm{PASC}^{33}$. In contrast, addition of $\mathrm{Xyl}_{6}$ did not give significant shifts in $g_{z}$ but did give perturbations in the $\left|A_{z}\right|$ value, with the appearance of superhyperfine coupling indicative of a second species different from that formed with Cell 6 . The EPR spectra of LsAA9A binding to $\mathrm{Xyl}_{6}$ and xylan are indicative of substrate binding to the enzyme (although binding of $\mathrm{Xyl}_{6}$ could be achieved only at high chloride concentrations), but without the chloride occupying the equatorial coordination position on the copper ion, revealing that these substrates drive an electronic state at the copper ion that is different to that of the other substrates. EPR perturbation was seen upon addition of $\mathrm{Cell}_{6}$ to $\mathrm{CvAA9A}$ but not with $\mathrm{Xyl}_{6}$, consistent with the observed activity on $\mathrm{Cell}_{6}$ and not $\mathrm{Xyl}_{6}$ (Fig. 7; Supplementary Fig. 15).

\section{Discussion}

Our understanding of the molecular basis for substrate binding and cleavage has been aided by the recent report of a crystal structure of $L s \mathrm{AA} 9 \mathrm{~A}$ in complex with $\mathrm{Cell}_{3}$ and Cell 6 , as well as biochemical and EPR data for LsAA9A on cellulosic substrates ${ }^{33}$. Here we have extended this biochemical, EPR and structural analysis by using a range of substrates as well as an additional related enzyme, $C v$ AA9A, to provide a better insight into substrate specificity.

Extensive probing of LsAA9A and CvAA9A substrate specificity showed that both cleave a range of cellulosic and noncellulosic substrates, some of which have been shown for other AA9s ${ }^{19}, 20,22,23,27,29,35,39-41$. We made a number of important observations. Notably, whereas LPMO activity on celluloseassociated xylan has been previously observed for $M t \mathrm{LPMO}^{23}$, we report activity of LsAA9A on xylo-oligosaccharides and isolated xylan; this may have important implications for the use of LPMOs in biotechnological contexts. We also observe that both LsAA9A and CvAA9A are able to cleave glycosidic bonds adjacent to mannosyl residues (Supplementary Fig. 6), which occur interspersed randomly with glucosyl residues in glucomannan, a biochemical observation supported by the LsAA9A:glucomannan oligosaccharide structure, which unambiguously shows a mannosyl residue at subsite +1 . We also noticed substrate-specific 
Table 2 Scaled crystallographic data statistics and refinement statistics

\begin{tabular}{|c|c|c|c|c|c|c|c|c|}
\hline & $\begin{array}{l}\text { LsAA9A: } \\
\text { Cell }_{5}\end{array}$ & $\begin{array}{l}\text { LsAA9A: } \\
\text { G4G4G3G }\end{array}$ & LsAA9A:Xyl ${ }_{3}$ & LsAA9A:Xyl ${ }_{4}$ & LsAA9A:Xyl ${ }_{5}$ & $\begin{array}{l}\text { LsAA9A: } \\
\mathrm{Xyl}_{5} \mathrm{Cu}(\mathrm{II})\end{array}$ & LsAA9A:GM & CVAA9A \\
\hline \multicolumn{9}{|l|}{ Data collection } \\
\hline Synchrotron & ESRF & MAX-lab & ESRF & ESRF & ESRF & ESRF & ESRF & MAX-lab \\
\hline Beamline & ID23-1 & 1911-2 & ID23-1 & ID23-1 & ID23-2 & ID23-2 & ID30-B & $1911-3$ \\
\hline Wavelength $(\lambda)$ & 0.97625 & 1.03841 & 0.97625 & 0.97625 & 0.87260 & 0.87260 & 0.96862 & 1.00000 \\
\hline Space group & $P 4,32$ & $P 4,32$ & $P 4,32$ & $P 4,32$ & $P 4,32$ & $P 4,32$ & $P 4,32$ & $P 1$ \\
\hline \multicolumn{9}{|l|}{ Cell dimensions } \\
\hline$a, b, c(\AA)$ & 126.30 & 124.91 & 125.18 & 125.01 & 125.35 & 125.30 & 125.29 & $\begin{array}{l}47.00,59.42, \\
115.45\end{array}$ \\
\hline$\alpha, \beta, \gamma\left(^{\circ}\right)$ & 90 & 90 & 90 & 90 & 90 & 90 & 90 & $\begin{array}{l}102.67,98.89, \\
89.54\end{array}$ \\
\hline \multirow[t]{2}{*}{ Resolution $(\AA)$} & $50.00-1.75$ & $50.00-2.00$ & $50.00-1.50$ & $44.20-1.59$ & $50.00-1.33$ & $50.00-1.90$ & $50.00-1.48$ & $50.00-1.90$ \\
\hline & $(1.80-1.75)^{a}$ & $(2.05-2.00)$ & $(1.54-1.50)$ & $(1.70-1.59)$ & $(1.36-1.33)$ & $(1.95-1.90)$ & $(1.52-1.48)$ & $(1.95-1.90)$ \\
\hline$R_{\text {meas }}$ & 0.118 (1.81) & $0.238(1.64)$ & 0.114 (1.67) & $0.097(1.84)$ & $0.098(2.62)$ & $0.289(1.58)$ & $0.133(3.24)$ & $0.124(0.828)$ \\
\hline$|/ \sigma|$ & $11.53(1.02)$ & $12.31(1.85)$ & $16.84(1.81)$ & $17.53(1.92)$ & $20.13(1.37)$ & $6.30(1.37)$ & $24.72(1.50)$ & $6.70(1.22)$ \\
\hline $\begin{array}{l}\text { Completeness } \\
(\%)\end{array}$ & $99.1(98.4)$ & 99.9 (99.9) & 99.7 (96.9) & 99.8 (100) & $100(100)$ & 99.8 (97.7) & $100(100)$ & $96.1(92.7)$ \\
\hline$C C(1 / 2)(\%)$ & $\begin{array}{l}99.7 \\
(40.4)\end{array}$ & 99.6 (49.8) & $99.8(51.6)$ & $99.9(61.8)$ & $100(51.6)$ & $98.8(62.5)$ & $100(57.7)$ & $99.1(64.3)$ \\
\hline Observed & 361,920 & 304,791 & 560,310 & 475,945 & $1,673,125$ & 234,494 & $3,982,032$ & 196,670 \\
\hline reflections & $(25,195)$ & $(19,136)$ & $(53,847)$ & $(87,679)$ & $(123,274)$ & $(16,918)$ & $(199,606)$ & $(10,957)$ \\
\hline Unique & 34,937 & $23,096(1680)$ & 36,460 & 45,264 & 77,258 & 27,034 (1914) & 105,966 & 90,992 \\
\hline reflections & $(2502)$ & & (3782) & $(8102)$ & $(5639)$ & & (7879) & (6493) \\
\hline Redundancy & $\begin{array}{l}10.36 \\
(10.07)\end{array}$ & $13.20(11.39)$ & $10.41(9.64)$ & $10.51(10.82)$ & 21.66 (21.88) & $8.67(8.84)$ & $37.59(25.33)$ & $2.16(1.69)$ \\
\hline \multicolumn{9}{|l|}{ Refinement } \\
\hline \multicolumn{9}{|l|}{ Resolution $(\AA)$} \\
\hline No. mol. ASU & 1 & 1 & 1 & 1 & 1 & 1 & 1 & 6 \\
\hline$R_{\text {work }} / R_{\text {free }}(\%)$ & $\begin{array}{l}18.15 / \\
21.45\end{array}$ & $17.30 / 21.09$ & $13.76 / 17.18$ & $16.02 / 17.55$ & $11.48 / 14.27$ & $16.40 / 20.71$ & $12.32 / 17.04$ & $19.79 / 24.44$ \\
\hline \multicolumn{9}{|l|}{ No. of atoms } \\
\hline Protein ${ }^{b}$ & 1821 & 1885 & 1884 & 1838 & 1882 & 1818 & 1869 & 10,673 \\
\hline Ligand/ion & 61 & 60 & 100 & 66 & 56 & 56 & 176 & 40 \\
\hline Water & 247 & 270 & 374 & 262 & 449 & 403 & 601 & 1041 \\
\hline \multicolumn{9}{|l|}{ B-factors } \\
\hline Protein ${ }^{b}$ & 32.1 & 23.9 & 17.8 & 25.0 & 18.0 & 18.4 & 22.8 & 26.2 \\
\hline Ligand/ion & 47.8 & 35.9 & 36.0 & 52.9 & 24.0 & 27.8 & 34.2 & 70.6 \\
\hline Water & 42.7 & 31.0 & 33.5 & 39.6 & 37.9 & 32.0 & 39.2 & 28.1 \\
\hline \multicolumn{9}{|l|}{ R.m.s. deviations } \\
\hline $\begin{array}{l}\text { Bond lengths } \\
(\AA)\end{array}$ & 0.0135 & 0.0183 & 0.0186 & 0.0174 & 0.0245 & 0.0167 & 0.026 & 0.0141 \\
\hline Bond angles $\left(^{\circ}\right)$ & 1.5018 & 1.9101 & 1.8982 & 1.7407 & 2.0278 & 1.7393 & 2.3372 & 1.7245 \\
\hline
\end{tabular}

oxidation profiles, namely that LsAA9A and CvAA9A cleaved small oligosaccharides using a C4-oxidising mechanism whereas they cleaved polysaccharides with both $\mathrm{C} 1$ - and $\mathrm{C} 4$-oxidising mechanisms in varying proportions. Assuming a copper-based oxidative species, the similar distances between both $\mathrm{C} 1$ and $\mathrm{C} 4$ axial protons and the active oxygen species, as noted in Frandsen et al. ${ }^{33}$, may allow slight differences in substrate binding to switch the $\mathrm{C}-\mathrm{H}$ bond that is closest to attack. Substrate binding differences may also subtly alter the electronics at the copper site, which potentially could also favour a specific oxidation site. Oxidation regioselectivity is therefore less likely to be a strong functional constraint. This is in agreement with the presence of $\mathrm{C} 1$ and $\mathrm{C} 4$ regiospecificity in several clades in the $\mathrm{N}$-terminal sequence similarity tree (Fig. 1). On the other hand, the tree enabled us successfully to predict that LsAA9A and CvAA9A might have similarities in having activity on a range of soluble substrates.

Our investigation highlighted many examples of the way in which substrate specificity and the site of attack on a polysaccharide is dictated by binding cleft interactions with the substrate. For example, LsAA9A's preference to cleave $\mathrm{Cell}_{4}$ into $\mathrm{Cell}_{2}$ (as shown for $N c \mathrm{LPMO}^{18}$ ), compared to the product profile of $\mathrm{CvAA9A}$ of $\mathrm{Cell}_{3}, \mathrm{Cell}_{2}$ and Glc (Supplementary Fig. 5), could be attributed to binding cleft interactions at the +2 subsite. This is because, though CvAA9A shares the same fold, active site co-ordination and overall structure with LsAA9A, it lacks all three of the subsite +2 substrate-binding residues in LsAA9A (Asn28, His66 and Asn67) (Supplementary Figs. 1 and 10). This suggests that, while $L s \mathrm{AA} 9 \mathrm{~A}$ binds $\mathrm{Cell}_{4}$ from subsite -2 to +2 , CvAA9A binds between subsites -3 and +1 .

The structural data provide a molecular rationale for how LsAA9A is able to catalyse the unexpected cleavage of mannosecontaining bonds. The LsAA9A:glucomannan oligosaccharide crystal structure shows the presence of a mannose (the $\mathrm{C} 2$ epimer of Glc), and essentially no Glc, at subsite +1 . Talose (C4 epimer of mannose) and galactose ( $\mathrm{C} 4$ epimer of Glc) arose from the reduction of $\mathrm{C} 4$-oxidised cleavage products (Supplementary Fig. 6), also suggesting both mannosyl and glucosyl occupation of 
a

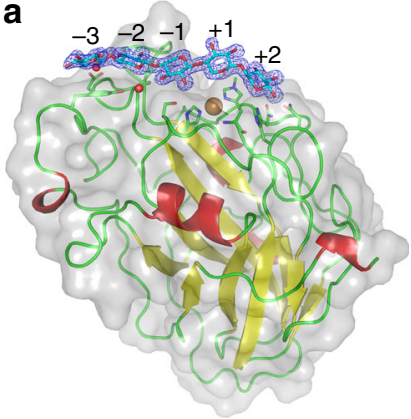

b

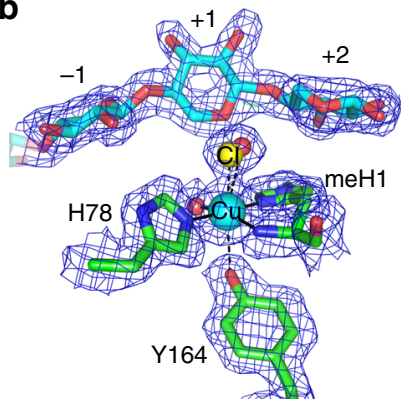

C

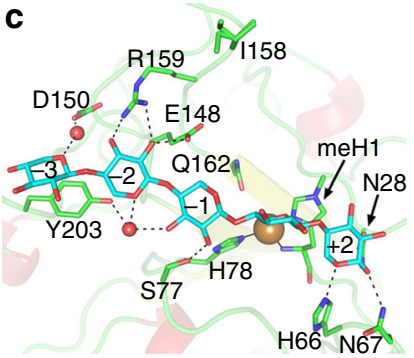

d

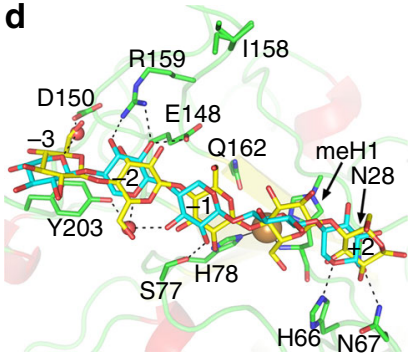

Fig. 6 Structure of the LsAA9A:xylo-oligosaccharide complex. a LsAA9A: $\mathrm{XyI}_{5}$ with bound substrate (in cyan) from subsite -3 to +2 . b Active site

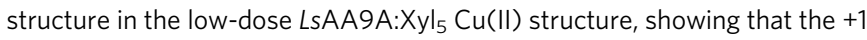
xylosyl unit does not directly interact with the enzyme or displace the axial ligand on the copper (modelled as chloride and water in 0.5:0.5 ratio). c Top-down view LsAA9A:Xyl (in cyan). d Top-down view $L s A A 9 A: X y I_{5}$ (in cyan) and $L s A A 9 A: C e l l_{5}$ (in yellow) shown for comparison. A $2 F_{\text {obs }}-F_{\text {calc }}$ electron density map is shown at $1 \sigma$ contour level in panels $\mathbf{a}$ and $\mathbf{b}$

subsite +1 . We have previously described that the glucosyl unit at the +1 subsite in $\mathrm{LsAA} \mathrm{AA} \mathrm{Cell}{ }_{3}$ and Cell complexes $^{33,38}$ interacts with MeHis1 through its $\beta$-face, and while glucose can make carbohydrate-aromatic stacking interactions ${ }^{42}$ through both faces of the pyranose ring, $\beta$-mannose is believed to have absolute preference for interactions through its $\alpha$-face due to its axial C2hydroxyl. Nonetheless, determination of the crystal structure of glucomannan fragments with LsAA9A confirmed that this type of interaction takes place, and the mannosyl residue at the +1 subsites interacts with $\mathrm{MeHis}$ through its $\beta$-face with an O5imidazole ring centre distance of $3.6 \AA$ (Fig. 5f) (compared to 3.4-3.5 $\AA$ for the cello-oligosaccharide complexes). No similar interactions could be found through a search in the PDB. We did not observe a mannosyl residue at the -1 subsite in the structure, but it would cause no steric clash and so could be readily accommodated (though it would cause the loss of a hydrogen bond interaction with Ser77).

Although we were unable to obtain a structure with xyloglucan oligosaccharides bound, our observation that both LsAA9A and CvAA9A cleaved xyloglucan DP14-18 oligomers with the sole unsubstituted backbone glucosyl residues at subsite +1 (XXX/ GXXXGol; as found for NcLPMO9C ${ }^{19}$ ) is consistent with the binding of xyloglucan's cellulosyl backbone being similar to the binding of cello-oligosaccharides. This would suggest that the LsAA9A could tolerate glucosyl residues with C6 xylosyl substitutions at subsites -1 or +2 , but not at +1 where the C6 hydroxymethyl group occludes the copper axial binding site, and displaces the axial water.

LsAA9A under the selected conditions degraded $\mathrm{Xyl}_{6}$ with about $1 / 100$ the efficiency as $\mathrm{Cell}_{6}$, while $C v \mathrm{AA} 9 \mathrm{~A}$ left $\mathrm{Xyl}_{6}$ essentially untouched at all conditions tested (Supplementary Fig. 9). The differences in key amino acids involved in defining the LsAA9A and CvAA9A subsites, particularly the +2 subsite (vide supra) (Supplementary Fig. 1 and Supplementary Fig. 10), are likely an important factor in LsAA9A's superior xylandegrading activity.

Our observation in LsAA9A complexes that MLG oligosaccharides were unable to bind with $\beta$ - $(1 \rightarrow 3)$-glucan bonds near the active site are consistent with our observation that both LsAA9A and CvAA9A favour the cleavage of cellulosyl regions in MLG.

Not all aspects of substrate specificity could be explained through binding cleft interactions. Rather, aspects of the specificity differences appear to be mechanistic in origin and relate to the reactivity of different substrates. The high activity, spectroscopy data and structures of LsAA9A and CvAA9A with $\beta-(1 \rightarrow$ 4)-glucan substrates leads us to suggest the effective oxidative mechanism deployed in these situations may be regarded as a 'canonical pathway'. It is clear, however, that LPMOs may also have other 'non-canonical pathway' mechanisms, as exemplified by the differences between binding, spectroscopy and structures of LsAA9A with $\mathrm{Xyl}_{6}$. The crystallographic and EPR data show that a chloride ion-an oxygen species mimic-is not recruited into the copper's equatorial binding site upon xylooligosaccharide substrate binding, as happens in the canonical mechanism described by Frandsen et al. ${ }^{33}$. The aldopentose nature of xylose categorically excludes the synergistic binding of saccharide ligand and molecular oxygen, which is brought about by a bridging 'pocket' water molecule between the C6-hydroxymethyl group of Glc and the amino terminus of the enzyme. This suggests a different oxidative mechanism may well be in operation for the cleavage of xylose-based substrates by LsAA9A. Indeed, as has already been proposed by Kjaergaard et al. ${ }^{43}$, activation of $\mathrm{O}_{2}$ by an AA9 from Thermoascus aurantiacus probably gives formation of a copper-bound superoxide or hydrosuperoxide $\left(\mathrm{HO}_{2}\right)$ through associative displacement of a superoxide anion by a water molecule through the axial coordination site on the copper ion. In particular, a superoxide ion bound to the copper in the axial position would be in position to cleave a saccharidic chain by direct attack. Such a mechanism is expected when the axial water molecule on the copper ion is not displaced by the binding of substrate, as is the case with the binding of $\mathrm{Xyl}_{5}$ to LsAA9A. From the low-dose LsAA9A:Xyl ${ }_{5}$ structure described herein, the axial ligand is clearly present on the copper ion, though it is best modelled as a mixture of chloride and water, and the Tyr-O distance $(2.86 \AA)$ is not shortened compared to the un-complexed low dose structure (2.72 $\AA$-PDB 5ACG). This is in contrast to the low dose $L s \mathrm{AA} 9 \mathrm{~A}: \mathrm{Cell}_{3}$ structure where the Tyr-O distance is $2.47 \AA$ (PDB 5ACF). Furthermore, the equatorial position in the low dose $L s \mathrm{AA} 9 \mathrm{~A}: \mathrm{Xyl}_{5}$ is occupied by a water molecule, not a chloride ion, as corroborated by the EPR spectroscopy. Thus, a mechanism by which a copper-bound superoxide is generated next to the substrate is possible within the LsAA9A-Xyl ${ }_{5}$ complex. Such a mechanism may be expected to be rate-independent on the redox potential of the reducing agent, since the rate-limiting step is likely to be hydrogen atom abstraction by the superoxide from the substrate rather than reductive cleavage of the $\mathrm{O}-\mathrm{O}$ bond. Therefore, the fact that the rate of cleavage of xylan and $\mathrm{Xyl}_{6}$ by $L s \mathrm{AA} 9 \mathrm{~A}$ is less dependent on reducing agent while the cleavage of the other substrates is strongly dependent (Fig. 4) illustrates that a different oxidative mechanism is in operation. Thus the extent of activity on certain substrates is a function of the oxidative species which can be formed at the copper ion which is-in turn-dependent on the substrate. This means that for some substrates the use of reducing agents with different potentials can profoundly affect apparent substrate specificity. But, more importantly, LPMOs appear to have more than one oxidative mechanism available for substrate cleavage, governed to some extent by the nature of the substrate-LPMO interaction. Indeed, the existence of multiple oxidative mechanisms for a 


\section{Table 3 Spin-Hamiltonian parameters (parallel region) for LsAA9A and CvAA9A in contact with substrates}

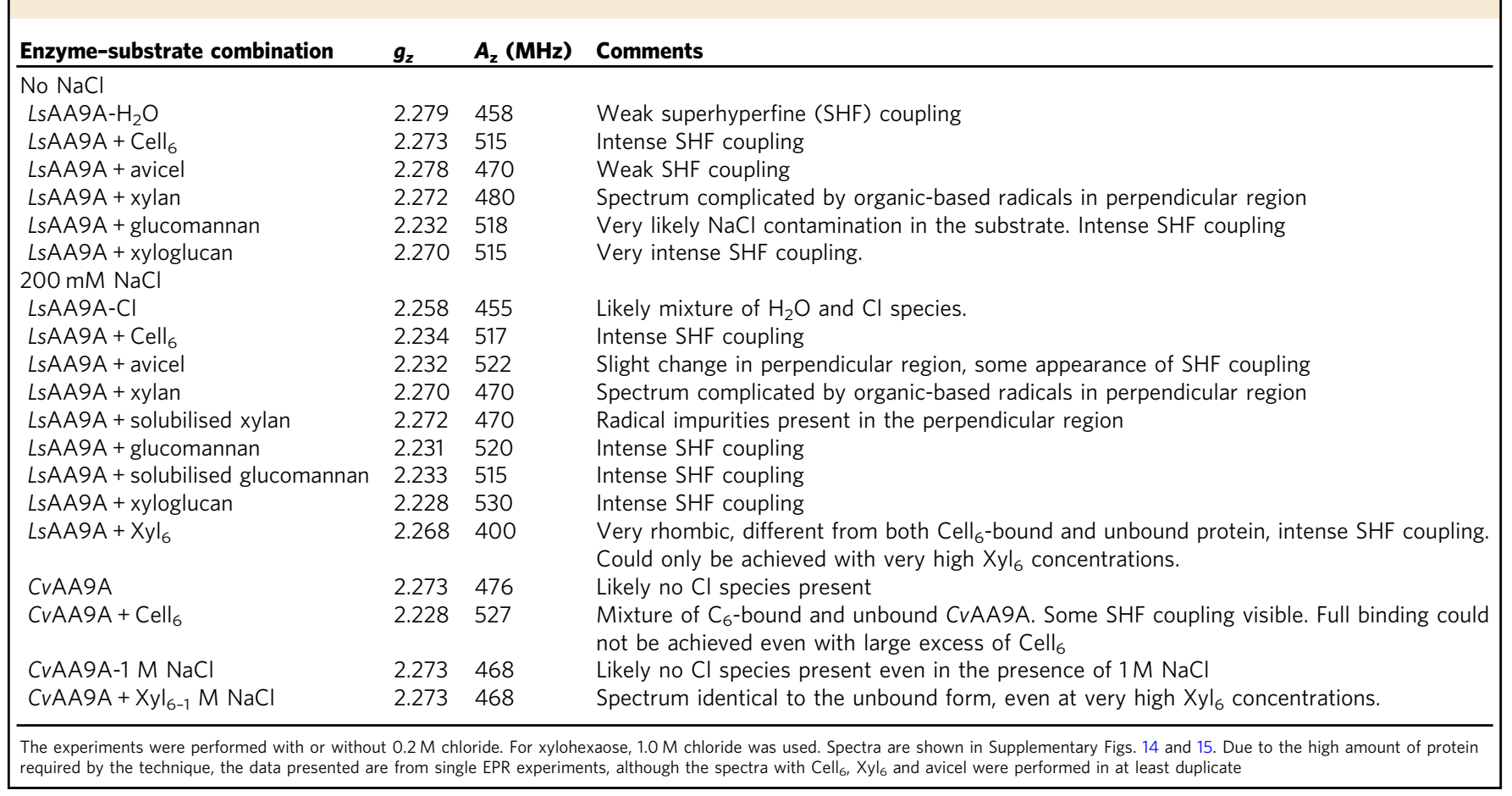
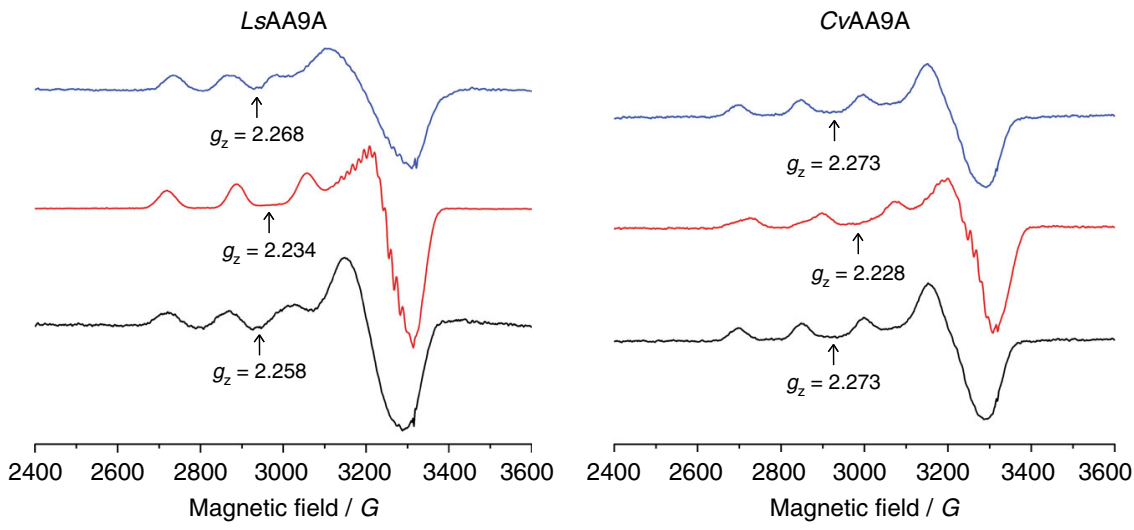

Fig. 7 X band cw EPR spectra of LsAA9A (left) and CVAA9A (right), $150 \mathrm{~K}$. Spectra were collected in the presence of $1 \mathrm{M} \mathrm{NaCl}$ (black), Cell 6 and $200 \mathrm{mM}$ $\mathrm{NaCl}$ (red), or $\mathrm{Xyl}_{6}$ and $1 \mathrm{M} \mathrm{NaCl}$ (blue). Spectra are representative of duplicate experiments

single LPMO is an intriguing contribution to the ongoing debate about LPMO mode of action.

Results obtained in this study broaden the known substrate specificity of AA9 LPMOs to include isolated xylan and xylo-oligosaccharides, and mannosyl-containing bonds within glucomannan. We further show that oxidation type $(\mathrm{C} 1 / \mathrm{C} 4)$ is influenced by substrate type, and in this work differed between oligo- and polysaccharides. This investigation into the molecular causes of AA9 LPMO substrate specificity demonstrated the existence of multiple influences. As with carbohydrate-acting hydrolases, for example, LPMO substrate specificity is dictated by binding cleft protein:carbohydrate interactions. But in addition, the fact that activity on some substrates is differentially responsive to reducing agent potential suggests that these carbohydrates do not properly activate the active site copper, and are cleaved through an alternative oxidative pathway. Combinations of canonical and non-canonical mechanisms greatly extend the range of potential substrates for LPMOs and offer new insight into their biochemical mode of action.

\section{Methods}

Phylogenetic tree. AA9 is a family with more than 6000 sequences listed in NCBI $\mathrm{nr}$ and JGI databases in 2016. Because of high variability in the N-terminal portion of LPMO amino-acid sequences, no significant global alignment of LPMOs can be obtained-thereby limiting global downstream phylogenetic analyses. We chose therefore to extract the highly variable $\mathrm{N}$-terminal half of these sequences (which includes two histidine residues involved in the coordination of the copper atom) for phylogenetic analysis as well as to limit the analysis to sequences that are closely related to each other and to those that have been biochemically characterized in the literature. We reduced the set of AA9 sequences to those that gave BLAST bitscores greater than or equal to a value of 200, using LsAA9A and TaAA9A as queries. A Jaccard distance matrix was compiled from BLAST bit scores and represented as a tree, built according to the principle of neighbor-joining method ${ }^{44}$ displaying the resulting 444 sequences (Fig. 1).

Protein production. The gene encoding LsAA9A was amplified from genomic DNA of Lentinus similis and the gene encoding CvAA9A was amplified from genomic DNA of Collariella virescens (formerly known as Chaetomium virescens). Both were cloned in $E$. coli using primers containing insertion sites for the vector pDau109 used for cloning. The fragments were then cloned into BamHl- and XhoIdigested pDau109 using an IN-FUSION Cloning Kit. Cloning of the genes into BamHl- and XhoI-digested pDau109 resulted in transcription of the recombinant 
enzymes encoding gene under the control of a NA2-tpi double promoter. The treated plasmids and inserts were transformed into One Shot TOP10F Chemically Competent $E$. coli cells (Invitrogen) according to the manufacturer's protocol, spread onto LB plates supplemented with $0.1 \mathrm{mg} / \mathrm{ml}$ ampicillin and incubated at $37^{\circ} \mathrm{C}$ overnight. Colonies of each transformation were cultivated in LB medium supplemented with $0.1 \mathrm{mg} / \mathrm{ml}$ ampicillin and plasmids were isolated using a QIAPREP Spin Miniprep Kit (Qiagen).

LsAA9A and CvAA9A was expressed in Aspergillus oryzae MT3568. Transformants producing the recombinant enzymes were inoculated in 21 of Dap$4 \mathrm{C}$ medium and incubated at $30^{\circ} \mathrm{C}$ for 4 days. Mycelia were removed by filtration, and the medium was collected for purification. Ammonium sulfate was added to the sterile filtered medium to a conductivity of $200 \mathrm{mSi} / \mathrm{cm}$ and the $\mathrm{pH}$ adjusted to 7.5. The broth was applied to a 50/15 Butyl Toyopearl column (Tosoh Biosciences) equilibrated with $25 \mathrm{mM}$ Tris, $1.5 \mathrm{M}$ ammonium sulfate, $\mathrm{pH}$ 7.5. The column was washed in the same buffer and eluted with a gradient to $25 \mathrm{mM}$ Tris, $\mathrm{pH} 7.5$. Fractions containing recombinant enzymes were combined and washed with milliQ water by ultrafiltration (10 kDa MWCO, PES filter, Sartorius) to a conductivity of $1.2 \mathrm{mSi} / \mathrm{cm}$. The $\mathrm{pH}$ was adjusted to 8.0 and applied to a $50 / 40 \mathrm{Q}$ Sepharose FF column (GE Healthcare) equilibrated with $20 \mathrm{mM}$ Tris, pH 8.0. The column was washed in the same buffer and the enzyme eluted with a gradient from 0 to $0.5 \mathrm{M}$ sodium chloride. Fractions containing LsAA9A were combined and concentrated by ultrafiltration using VIVASPIN $20(10 \mathrm{kDa}$ MWCO) spin concentrators.

Enzyme assays. Apo-LsAA9A and apo- $C v \mathrm{AA} 9 \mathrm{~A}$ were pre-incubated for $0.5-1 \mathrm{~h}$ at $5{ }^{\circ} \mathrm{C}$ in 0.9 stoichiometric $\mathrm{Cu}(\mathrm{II})\left(\mathrm{NO}_{3}\right)_{2}$ immediately before enzyme reactions. AA9 enzyme reactions on oligosaccharides were in $10 \mu \mathrm{l}$ containing $5 \mathrm{nmol}$ oligosaccharide, $100 \mathrm{mM}$ ammonium formate $\mathrm{pH} 6, \pm 4 \mathrm{mM}$ ascorbate, pyrogallol or cysteine, \pm 5 pmol LsAA9A or TaAA9A and were incubated at $20^{\circ} \mathrm{C}$ for $4 \mathrm{~h}$. Xyloglucan endoglucanase (XEG) reactions were in $10 \mu \mathrm{l}$ containing $5 \mathrm{nmol}$ oligosaccharide, $100 \mathrm{mM}$ ammonium formate $\mathrm{pH} 6, \pm 10 \mu \mathrm{mol}$ GH5 XEG and were incubated at $20^{\circ} \mathrm{C}$ for $4 \mathrm{~h}$. Oligosaccharides were purchased from Megazyme (see also following section). In general, enzyme reactions on polysaccharides were in $100 \mu \mathrm{l}$ containing $0.5 \%(\mathrm{w} / \mathrm{v})$ polysaccharide, $100 \mathrm{mM}$ ammonium formate $\mathrm{pH} 6$, $\pm 4 \mathrm{mM}$ ascorbate, pyrogallol or cysteine, \pm 63 pmol LPMO, and were incubated at $20{ }^{\circ} \mathrm{C}$ for $16 \mathrm{~h}$. Avicel cellulose was purchased from Sigma-Aldrich, UK; barley beta-glucan medium viscosity (mixed-linkage glucan), konjac glucomannan, tamarind xyloglucan, birchwood xylan, corn starch and laminarin were purchased from Megazyme, Ireland; squid-pen $\beta$-chitin was a kind gift from Dominique Gillet of Mahtani Chitosan. Phosphoric acid-swollen cellulose (PASC) was prepared by making a slurry of $1 \mathrm{~g}$ Avicel cellulose (Sigma-Aldrich) with $3 \mathrm{ml} \mathrm{H} 2 \mathrm{O}$ before adding $30 \mathrm{ml}$ ice-cold phosphoric acid and incubating at $0{ }^{\circ} \mathrm{C}$ for $1 \mathrm{~h}$. The cellulose was then washed numerous times with water until the flowthrough had a neutral $\mathrm{pH}$ before use in reactions. Mixed-linkage glucan, glucomannan, xyloglucan, xylan, starch and laminarin were boiled for $5 \mathrm{~min}$ to make solubilized $1 \%(\mathrm{w} / \mathrm{v})$ stock solutions before reactions. To aid solubilisation where necessary, water was added to a methanol: polysaccharide slurry before boiling, which improved dispersion throughout the water. Reactions were routinely stopped by addition of three reaction volumes of $96 \%(\mathrm{v} / \mathrm{v})$ ethanol before precipitation of the undigested substrates, and separation of the reaction products for further analysis. For polysaccharide analysis by carbohydrate electrophoresis (PACE), reaction products and oligosaccharide standards (Megazyme) were reductively aminated with 8-aminonaphthalene-1,3,6-trisulfonic acid (ANTS; Invitrogen, http://www.lifetechnologies. com) and separated by acrylamide gel electrophoresis. In all cases, an Hoefer SE 660 vertical slab gel electrophoresis apparatus (Amersham, Buckinghamshire, UK) was used with $24-\mathrm{cm}$ plates, $0.75-\mathrm{mm}$ spacer, and well of width $0.25 \mathrm{~cm}$. Standard glass or low-fluorescence Pyrex plates were used. Electrophoresis was performed at $10^{\circ} \mathrm{C}$ in all cases. High concentration gel PACE was performed using a $192 \mathrm{mM}$ glycine, $25 \mathrm{mM}$ Tris, $\mathrm{pH}$ 8.5, running buffer. The gel contents were as follows: resolving gels, $37.5 \mathrm{ml} 40 \%$ (w/v) acrylamide, $12.5 \mathrm{ml} 375 \mathrm{mM}$ Tris- $\mathrm{HCl}$ buffer, $\mathrm{pH}$ 8.8, $100 \mu \mathrm{l} 10 \%$ ammonium persulfate, $50 \mu \mathrm{l}$ tetramethylethylenediamine (TEMED); stacking gels, $2 \mathrm{ml} \mathrm{40 \%} \mathrm{(w/v)} \mathrm{acrylamide,} 2.5 \mathrm{ml} 375 \mathrm{mM}$ Tris- $\mathrm{HCl}$ buffer, $\mathrm{pH} 8.8,5.4 \mathrm{ml}$ water, $100 \mu \mathrm{l} 10 \%$ ammonium persulfate, $10 \mu \mathrm{l}$ TEMED. Electrophoresis was carried out at $100 \mathrm{~V}$ for $30 \mathrm{~min}, 500 \mathrm{~V}$ for $30 \mathrm{~min}$ and $1000 \mathrm{~V}$ for 180-210 min, and gels were then visualized with a G-box (Syngene) equipped with a short pass detection filter $(500-600 \mathrm{~nm})$ and long-wave UV tubes $(365 \mathrm{~nm}$ emission). Low concentration gel PACE was performed using a 0.1 M Tris-borate $\mathrm{pH} 8.2$ buffer system. The gel contents were as follows: $20 \%(\mathrm{w} / \mathrm{v})$ polyacrylamide gel contained $0.5 \%(\mathrm{w} / \mathrm{v}) \mathrm{N}, \mathrm{N} 9$-methylenebisacrylamide with a stacking gel $(2 \mathrm{~cm})$ of $8 \%(\mathrm{w} / \mathrm{v})$ polyacrylamide and $0.2 \%(\mathrm{w} / \mathrm{v}) \mathrm{N}, \mathrm{N}$ 9-methylenebisacrylamide. The samples were electrophoresed initially at $200 \mathrm{~V}$ for $20 \mathrm{~min}$ and then at $1000 \mathrm{~V}$ for $90 \mathrm{~min}$. All experiments were carried out at least in triplicate. See Supplementary Fig. 16 for uncropped gels scans.

Sodium borohydride reducing agent experiments were performed by addition of ammonia to a concentration of $2 \mathrm{M}$ to reaction mixtures. After incubation for 5 mins, $1 / 20$ solution volumes of $10 \%(\mathrm{w} / \mathrm{v}) \mathrm{NaBH}_{4} 2 \mathrm{M}$ ammonia were added, before incubation for $16 \mathrm{~h}$ at $25^{\circ} \mathrm{C}$. Samples were dried in vacuo and redissolved in, and redried from, $100 \mu \mathrm{l} 10 \%(\mathrm{v} / \mathrm{v})$ acetic acid $90 \%(\mathrm{v} / \mathrm{v})$ methanol, five times. HPAEC was performed on a CarboPac PA1 column (Dionex) with injections of 20 $\mu \mathrm{l}$ and elution at $0.4 \mathrm{ml} \mathrm{min}-1$. The elution profile was: $0-3 \mathrm{~min}, 10 \mathrm{mM} \mathrm{NaOH}$ (isocratic); 3-6 min, $10 \rightarrow 1 \mathrm{mM} \mathrm{NaOH}$ (linear gradient); 6-19 min, $1 \mathrm{mM} \mathrm{NaOH}$ (isocratic); $19-37 \mathrm{~min}, 45 \mathrm{mM} \mathrm{NaOH}, 225 \mathrm{mM}$ sodium acetate (isocratic). A pulsed amperometric detector $(\mathrm{PAD})$ with a gold electrode was used. $\mathrm{PAD}$ response was calibrated using markers $(500 \mathrm{pmol})$.

X-ray crystallography and PDB database searches. All crystallization trials were set up in MRC two-well plates at room temperature using an Oryx-8 robot (Douglas Instrument). Crystals were obtained by sitting-drop vapour diffusion technique in drops of $0.3-0.5 \mu \mathrm{l}$ with a reservoir volume of $100 \mu$ l. Pre-incubation with 1-2 mM Cu(II) acetate for 30-60 min was carried out for all crystallization trials. Crystallization and post-crystallization experimental details are shown in Supplementary Table 4. Crystals were cryocooled in liquid nitrogen and all datasets were collected at cryogenic temperatures $(100 \mathrm{~K})$ at either the MX beamlines I9112/I911-3 at MAX-lab in Lund, Sweden, or at the MX beamlines ID23-1, ID23-2 or ID30-B at ESRF, Grenoble, France (Table 2). All crystals were obtained by vapour diffusion technique set up in sitting-drop MRC plates, with a reservoir volume of $100 \mu \mathrm{l}$ and at room temperature using an Oryx-8 robot (Douglas Instrument). All data were collected at cryogenic temperatures $(100 \mathrm{~K})$ after cryocooling the crystals in liquid nitrogen. Oligosaccharide substrates used for soaking were purchased from Megazyme (MLG (G4G4G3G and G4G3G4G), xylotriose $\left(\mathrm{Xyl}_{3}\right.$ ), xylotetraose $\left(\mathrm{Xyl}_{4}\right)$, xylopentaose $\left(\mathrm{Xyl}_{5}\right)$, xyloglucan heptasaccharide (XXXG), cellotriose $\left(\mathrm{Cell}_{3}\right)$ or provided by Novozymes A/S (cellopentaose $\left(\mathrm{Cell}_{5}\right)$ ). Data were initially collected on crystals soaked with G4G4G3G (LsAA9A:G4G4G3G; PDB 5NLR), Xyl 3 (LsAA9A:Xyl ${ }_{3}$; PDB 5NLQ), $\mathrm{Xyl}_{4}$ (LsAA9A:Xyl ${ }_{4}$; PDB 5NLP) and Cell ${ }_{5}$ (LsAA9A: $\mathrm{Cell}_{5}$; PDB 5NLS). On a crystal soaked with $\mathrm{Xyl}_{5}$ a data set with reduced X-ray dose ((LsAA9A:Xyl $1_{5} \mathrm{Cu}(\mathrm{II})$; PDB $5 \mathrm{NLN} ; 40$ frames of $5.7 \%$ transmission, $0.05 \mathrm{~s}$ exposure/frame, $1^{\circ}$ oscillation with a beamsize of $\left.10 \times 10 \mu \mathrm{m}\right)$ was collected using helical collection to minimize photoreduction of the active site copper. Subsequently, on similar crystals another full dose data set was collected to high resolution (LsAA9A $\mathrm{Xyl}_{5}$; PDB 5NLO). Ladders of glucomannan (GM), from konjac, and of xyloglucan (XG), from tamarind, were prepared by partial acid hydrolysis (20-200 mM TFA for $20 \mathrm{~min}$ at $120^{\circ} \mathrm{C}$ ) of polysaccharide substrates purchased from Megazyme. Hydrolyzed products were isolated using ethanol precipitation to remove the remaining polysaccharides. The oligosaccharides were dried thoroughly using a SpeedVac. Data were collected on crystals soaked in GM (LsAA9A:GM; PDB $5 \mathrm{NKW}$ ) or XG stock solutions (in $3.8 \mathrm{M} \mathrm{NaCl}, 0.1 \mathrm{M}$ citric acid pH 5.5). Crystals were also soaked in the presence of $0.3 \mathrm{M} \mathrm{XXXG}$, and up to $1.2 \mathrm{M}$ of XG oligosaccharide purchased from Megazyme (consisting primarily of XXXGXXXG, see Courtade et al. ${ }^{21}$ ). No complex structures were obtained from any of the crystals soaked with XG substrates, either because no binding was observed or only cellooligosaccharides were bound (presumably because acid hydrolysis caused debranching). A stereo figure for typical density in the lowest resolution LsAA9A: oligosaccharide complex is shown in Supplementary Fig. 12.

CvAA9A was deglycosylated in $20 \mathrm{mM}$ MES, pH $6.0,125 \mathrm{mM} \mathrm{NaCl}$ by incubation with $\sim 0.03$ units per mg CvAA9A of endoglycosidase $\mathrm{H}$ from (Roche Diagnostics, 11643053001), and then buffer exchanged to $20 \mathrm{mM} \mathrm{Na}$-acetate $\mathrm{pH}$ 5.5. Intergrown crystals were initially obtained in an index screen in conditions of $1.5-2.0 \mathrm{M}\left(\mathrm{NH}_{4}\right)_{2} \mathrm{SO}_{4}$ (and in some cases $\left.0.1 \mathrm{M} \mathrm{NaCl}\right)$ in pH $6.5-8.5(0.1 \mathrm{M}$ of either Bis-Tris, HEPES or Tris). The crystals diffracted to 2.0-3.5 $\AA$ resolution but were multiple. Crystal conditions were optimized in a range of $1.2 \mathrm{M}-2.6 \mathrm{M}$ $\left(\mathrm{NH}_{4}\right)_{2} \mathrm{SO}_{4}( \pm 0.1 \mathrm{M} \mathrm{NaCl})$ in $\mathrm{pH}$ 6.5-8.5, which produced crystal plates suitable for mounting. A data set collected at I911-3 on a crystal grown in $0.1 \mathrm{M}$ Bis-Tris pH 6.5, $2.0 \mathrm{M}\left(\mathrm{NH}_{4}\right)_{2} \mathrm{SO}_{4}$ could be processed in $P 2_{1}$ to $2.5 \AA$. A preliminary CvAA9A structure with four molecules in the asymmetric unit was solved by Molecular Replacement using MOLREP with modified coordinates of the highresolution structure of LsAA9A (PDB 5ACH), which is $41 \%$ identical, as a model and refined isotropically to an $R_{\text {free }}$ of $32 \%$. From another data set (collected on a crystal grown in presence of $0.1 \mathrm{M} \mathrm{NaCl}$; Supplementary Table 4) a structure solved (using the preliminary one) with six molecules in the asymmetric unit could be fully modelled and refined resulting in the complete CvAA9A structure (PDB 5NLT; Table 2). The six molecules in the asymmetric unit are very similar (average RMSDs of $0.08 \AA$ ). The density of MeHis1 is less clear in chains C and F, and in particular methylation is not as obvious in all chains. Soaks (with $1.2 \mathrm{M} \mathrm{Cell}_{3}$ ) of CvAA9A were also prepared. Data were collected to $2.1 \AA$ and the electron density showed a Cell ${ }_{3}$ molecule, which however was not bound at the active site. Soaks with $\mathrm{Cell}_{6}$ damaged the crystals.

Each data set was processed using $\mathrm{XDS}^{45}$ (the resolution cutoff was chosen on the basis of a CC1/2 around 50\%) and subsequently scaled using XSCALE. Refmac $5^{46}$ was used for restrained refinement of the structures in which LsAA9A: $\mathrm{Xyl}_{5}$ was refined anisotropically, while $L s \mathrm{AA} 9 \mathrm{~A}: \mathrm{Xyl}_{3}$ was refined anisotropically for protein atoms and isotropically for all other atoms. All other structures were refined isotropically for all atoms. For LsAA9A:G4G4G3G the structure was best modelled by the G4G4G portion of the substrate bound mainly in subsite -1 to +2 ( $80 \%$ occupancy) and with a minor conformation occupying subsite -2 to $+1(20 \%$ occupancy). Near subsite, -2 a number of water molecules were modelled with $80 \%$ occupancy. Ligands and structures were modelled in $\mathrm{COOT}^{47}$ and validated using MolProbity (within COOT) and Procheck (CCP4 suite), which reported Ramachandran plots with $99 \%$ of residues in allowed regions for all structures. Scaled data statistics and refinement statistics are summarized in Table 2.

To identify potential stacking interactions of the $\beta$-face of $\beta$-mannose with His, the PDB database was searched with Glyvicinity ${ }^{48}$. First all protein/ $\beta$-mannose 
interactions within a distance cut-off of $4.0 \AA$ for structures determined at a resolution better than $3.0 \AA$ were identified. Among these, only two structures were found where the interactions involved His residues and the pyranose O5. The interactions between the imidazole and the pyranose rings were side by side or almost perpendicular, and thus not comparable with the +1 subsite interactions of the LsAA9A complexes.

EPR spectroscopy. Continuous wave (cw) X-band frozen solution EPR spectra of $0.2-0.3 \mathrm{mM}$ solution of $L s \mathrm{AA} 9 \mathrm{~A}$ or $C v \mathrm{AA} 9 \mathrm{~A}$ (in $10 \% \mathrm{v} / \mathrm{v}$ glycerol) at $\mathrm{pH} 6.0(50$ $\mathrm{mM}$ sodium phosphate buffer with or without addition of $200 \mathrm{mM} \mathrm{NaCl}$ or $20 \mathrm{mM}$ MES buffer, $200 \mathrm{mM} \mathrm{NaCl}$ ) and $165 \mathrm{~K}$ were acquired on a Bruker EMX spectrometer operating at $\sim 9.30 \mathrm{GHz}$, with modulation amplitude of $4 \mathrm{G}$, modulation frequency $100 \mathrm{kHz}$ and microwave power of $10.02 \mathrm{~mW}$ (three scans). Avicel cellulose, konjac glucomannan, tamarind xyloglucan and birchwood xylan were added to the EPR tube containing the protein as solids. Alternatively, glucomannan and xylan were heated until dissolution (ca. $2 \mathrm{~min}$ ) to make solubilized $1 \%(\mathrm{w} / \mathrm{v})$ stock solutions in water, which were then used for addition of excess polysaccharide to LsAA9A. Cellohexaose and xylohexaose were added to the protein solution either from stock solutions in water or as a solid up to 60 - or 150 -fold excess, respectively. For the experiments in the presence of xylohexaose, additional $\mathrm{NaCl}$ was added to the protein alone or the protein:Xyl ${ }_{6}$ mixture from a $5 \mathrm{M}$ stock solution. Due to the high amount of protein required by the technique, the data presented are from single EPR experiments, although the spectra with $\mathrm{Cell}_{6}, \mathrm{Xyl}_{6}$ and avicel were performed in at least duplicate.

Spectral simulations were carried out using EasySpin 5.0.3 ${ }^{49}$ integrated into MATLAB R2016 ${ }^{50}$ software on a desktop PC. Simulation parameters are given in Table 3. $g_{z}$ and $\left|A_{z}\right|$ values were determined accurately from the absorptions at low field. It was assumed that $g$ and $A$ tensors were axially coincident.

Data availability. Protein Data Bank: Atomic coordinates and structure factors for the reported crystal structures were deposited under accession codes 5NLT (CvAA9A), 5NLS (LsAA9A-Cell 5 ), 5NLR (LsAA9A-G4G4G3G), 5NKW (LsAA9AGM), 5NLQ (LsAA9A-Xyl $\left.{ }_{3}\right), 5 \mathrm{NLP}\left(L s \mathrm{AA} 9 \mathrm{~A}-\mathrm{Xyl}_{4}\right), 5 \mathrm{NLO}\left(\mathrm{LsAA} \mathrm{AA}-\mathrm{Xyl}_{5}\right)$ and 5NLN ( $L s A A 9 A-\mathrm{Xyl}_{5}-\mathrm{Cu}_{\mathrm{II}}$ ), GenBank: Sequence data for CvAA9A were deposited under accession code KY884985. Raw EPR data are available on request through the Research Data York (DOI: 10.15124/5810c962-148c-4328-ab92-895e2dae4d3c). The data that support the findings of this study are available from the corresponding author upon request.

Received: 23 April 2017 Accepted: 29 August 2017

Published online: 20 October 2017

\section{References}

1. Carroll, A. \& Somerville, C. Cellulosic biofuels. Annu. Rev. Plant. Biol. 60, 165-182 (2009).

2. Perlack, R. D. \& Stokes, B. J. U.S. Billion-Ton Update: Biomass Supply for a Bioenergy and Bioproducts Industry. Technical Report ORNL/TM-2011/224, 227 (US Department of Energy, Oak Ridge National Laboratory: Oak Ridge, TN, 2011).

3. Marriott, P. E., Gomez, L. D. \& McQueen-Mason, S. J. Unlocking the potential of lignocellulosic biomass through plant science. New. Phytol. 209, 1366-1381 (2016).

4. Simmons, T. J. et al. Folding of xylan onto cellulose fibrils in plant cell walls revealed by solid-state NMR. Nat. Commun. 7, 13902 (2016).

5. Correa, T. L. R., dos Santos, L. V. \& Pereira, G. A. G. AA9 and AA10: from enigmatic to essential enzymes. Appl. Microbiol. Biotechnol. 100, 9-16 (2016).

6. Muller, G., Varnai, A., Johansen, K. S., Eijsink, V. G. H. \& Horn, S. J. Harnessing the potential of LPMO-containing cellulase cocktails poses new demands on processing conditions. Biotechnol. Biofuels. 8, 817 (2015).

7. Hemsworth, G. R., Henrissat, B., Davies, G. J. \& Walton, P. H. Discovery and characterization of a new family of lytic polysaccharide monooxygenases. Nat. Chem. Biol. 10, 122-126 (2014).

8. Cantarel, B. L. et al. The carbohydrate-active EnZymes database (CAZy): an expert resource for glycogenomics. Nucleic Acids Res. 37, D233-D238 (2009).

9. Lombard, V., Ramulu, H. G., Drula, E., Coutinho, P. M. \& Henrissat, B. The carbohydrate-active enzymes database (CAZy) in 2013. Nucleic Acids Res. 42, D490-D495 (2014).

10. Vu, V. V., Beeson, W. T., Span, E. A., Farquhar, E. R. \& Marletta, M. A. A family of starch-active polysaccharide monooxygenases. Proc. Natl Acad. Sci. USA 111, 13822-13827 (2014)

11. Lo Leggio, L. et al. Structure and boosting activity of a starch-degrading lytic polysaccharide monooxygenase. Nat. Commun. 6, 5961 (2015).

12. Quinlan, R. J. et al. Insights into the oxidative degradation of cellulose by a copper metalloenzyme that exploits biomass components. Proc. Natl Acad. Sci. USA 108, 15079-15084 (2011)

13. Hemsworth, G. R., Johnston, E. M., Davies, G. J. \& Walton, P. H. Lytic polysaccharide monooxygenases in biomass conversion. Trends Biotechnol. 33, 747-761 (2015).
14. Vaaje-Kolstad, G., Horn, S. J., van Aalten, D. M. F., Synstad, B. \& Eijsink, V. G. $\mathrm{H}$. The non-catalytic chitin-binding protein CBP21 from Serratia marcescens is essential for chitin degradation. J. Biol. Chem. 280, 28492-28497 (2005).

15. Merino, S. T. \& Cherry, J. Progress and challenges in enzyme development for Biomass utilization. Adv. Biochem. Eng. Biotechnol. 108, 95-120 (2007).

16. Harris, P. V. et al. Stimulation of lignocellulosic biomass hydrolysis by proteins of glycoside hydrolase family 61: structure and function of a large, enigmatic family. Biochemistry 49, 3305-3316 (2010).

17. Vaaje-Kolstad, G. et al. An oxidative enzyme boosting the enzymatic conversion of recalcitrant polysaccharides. Science 330, 219-222 (2010).

18. Isaksen, T. et al. A C4-oxidizing lytic polysaccharide monooxygenase cleaving both cellulose and cello-oligosaccharides. J. Biol. Chem. 289, 2632-2642 (2014).

19. Agger, J. W. et al. Discovery of LPMO activity on hemicelluloses shows the importance of oxidative processes in plant cell wall degradation. Proc. Natl Acad. Sci. USA 111, 6287-6292 (2014).

20. Bennati-Granier, C. et al. Substrate specificity and regioselectivity of fungal AA9 lytic polysaccharide monooxygenases secreted by Podospora anserina. Biotechnol. Biofuels. 8, 90 (2015).

21. Courtade, G. et al. Backbone and side-chain H-1, C-13, and (15) N chemical shift assignments for the apo-form of the lytic polysaccharide monooxygenase NcLPMO9C. Biomol. NMR Assign. 10, 277-280 (2016).

22. Jagadeeswaran, G., Gainey, L., Prade, R. \& Mort, A. J. A family of AA9 lytic polysaccharide monooxygenases in Aspergillus nidulans is differentially regulated by multiple substrates and at least one is active on cellulose and xyloglucan. Appl. Microbiol. Biotechnol. 100, 4535-4547 (2016).

23. Frommhagen, M. et al. Discovery of the combined oxidative cleavage of plant xylan and cellulose by a new fungal polysaccharide monooxygenase. Biotechnol. Biofuels. 8, 101 (2015).

24. Levasseur, A., Drula, E., Lombard, V., Coutinho, P. M. \& Henrissat, B. Expansion of the enzymatic repertoire of the CAZy database to integrate auxiliary redox enzymes. Biotechnol. Biofuels. 6, 41 (2013).

25. Yakovlev, I. et al. Substrate-specific transcription of the enigmatic GH61 family of the pathogenic white-rot fungus Heterobasidion irregulare during growth on lignocellulose. Appl. Microbiol. Biotechnol. 95, 979-990 (2012).

26. Poidevin, L. et al. Comparative analyses of Podospora anserina secretomes reveal a large array of lignocellulose-active enzymes. Appl. Microbiol. Biotechnol. 98, 7457-7469 (2014).

27. Frommhagen, M. et al. Lytic polysaccharide monooxygenases from Myceliophthora thermophila C1 differ in substrate preference and reducing agent specificity. Biotechnol. Biofuels. 9, 186 (2016).

28. Kracher, D. et al. Extracellular electron transfer systems fuel cellulose oxidative degradation. Science 352, 1098-1101 (2016).

29. Borisova, A. S. et al. Structural and functional characterization of a lytic polysaccharide monooxygenase with broad substrate specificity. J. Biol. Chem. 290, 22955-22969 (2015)

30. Frandsen, K. E. H. \& Lo Leggio, L. Lytic polysaccharide monooxygenases: a crystallographer's view on a new class of biomass-degrading enzymes. IUCrJ 3 , 448-467 (2016).

31. Vaaje-Kolstad, G., Forsberg, Z., Loose, J. S., Bissaro, B. \& Eijsink, V. G. Structural diversity of lytic polysaccharide monooxygenases. Curr. Opin. Struct. Biol. 44, 67-76 (2017).

32. Davies, G. J., Wilson, K. S. \& Henrissat, B. Nomenclature for sugar-binding subsites in glycosyl hydrolases. Biochem. J. 321(Pt 2), 557-559 (1997).

33. Frandsen, K. E. H. et al. The molecular basis of polysaccharide cleavage by lytic polysaccharide monooxygenases. Nat. Chem. Biol. 12, 298-303 (2016).

34. Walton, P. H. \& Davies, G. J. On the catalytic mechanisms of lytic polysaccharide monooxygenases. Curr. Opin. Chem. Biol. 31, 195-207 (2016).

35. Cannella, D. et al. Light-driven oxidation of polysaccharides by photosynthetic pigments and a metalloenzyme. Nat. Commun. 7, 11134 (2016).

36. Lenfant, $\mathrm{N}$. et al. A bioinformatics analysis of 3400 lytic polysaccharide oxidases from family AA9. Carbohydr. Res. 448, 166-174 (2017).

37. Loose, J. S. M. et al. Activation of bacterial lytic polysaccharide monooxygenases with cellobiose dehydrogenase. Protein. Sci. 25, 2175-2186 (2016).

38. Frandsen, K. E. H., Poulsen, J. N., Tandrup, T. \& Lo Leggio, L. Unliganded and substrate bound structures of the cellooligosaccharide active lytic polysaccharide monooxygenase LsAA9A at low pH. Carbohydr. Res. 448, 187-190 (2017).

39. Kojima, Y. et al. A lytic polysaccharide monooxygenase with broad xyloglucan specificity from the brown-rot fungus Gloeophyllum trabeum and its action on cellulose-xyloglucan complexes. Appl. Environ. Microbiol. 82, 6557-6572 (2016).

40. Nekiunaite, L., Arntzen, M. O., Svensson, B., Vaaje-Kolstad, G. \& Abou Hachem, M. Lytic polysaccharide monooxygenases and other oxidative enzymes are abundantly secreted by Aspergillus nidulans grown on different starches. Biotechnol. Biofuels. 9, 187 (2016).

41. Fanuel, M. et al. The Podospora anserina lytic polysaccharide monooxygenase $\mathrm{PaLPMO}$ 9H catalyzes oxidative cleavage of diverse plant cell wall matrix glycans. Biotechnol. Biofuels. 10, 63 (2017). 
42. Asensio, J. L., Arda, A., Canada, F. J. \& Jimenez-Barbero, J. Carbohydratearomatic interactions. Acc. Chem. Res. 46, 946-954 (2013).

43. Kjaergaard, C. H. et al. Spectroscopic and computational insight into the activation of O-2 by the mononuclear $\mathrm{Cu}$ center in Polysaccharide monooxygenases. Proc. Natl Acad. Sci. USA 111, 8797-8802 (2014).

44. Lefort, V., Desper, R. \& Gascuel, O. FastME 2.0: a comprehensive, accurate, and fast distance-based phylogeny inference program. Mol. Biol. Evol. 32, 2798-2800 (2015).

45. Kabsch, W. XDS. Acta Crystallogr. D66, 125-132 (2010).

46. Vagin, A. A. et al. REFMAC5 dictionary: organization of prior chemical knowledge and guidelines for its use. Acta Crystallogr. D60, 2184-2195 (2004).

47. Emsley, P. \& Cowtan, K. Coot: model-building tools for molecular graphics. Acta Crystallogr. D60, 2126-2132 (2004).

48. Rojas-Macias, M. A. \& Lutteke, T. Statistical analysis of amino acids in the vicinity of carbohydrate residues performed by GlyVicinity. Methods Mol. Biol. 1273, 215-226 (2015).

49. Stoll, S. \& Schweiger, A. EasySpin, a comprehensive software package for spectral simulation and analysis in EPR. J. Magn. Reson. 178, 42-55 (2006).

50. MATLAB. MATLAB and Statistics Toolbox Release (The MathWorks, Inc., Natick, MA, USA, 2014).

\section{Acknowledgements}

We thank MAX-lab, Sweden and the European Synchrotron Radiation Facility (ESRF), France, for synchrotron beamtime and assistance. Travel to synchrotrons was supported by the Danish Ministry of Higher Education and Science through the Instrument Center DANSCATT and the European Community's Seventh Framework Programme (FP7/ 2007-2013) under BioStruct-X (grant agreement 283570). This work was supported by the UK Biotechnology and Biological Sciences Research Council (grant numbers BB/ L000423/1 to P.D. and P.H.W., and BB/L021633/1 to P.H.W.) and the Danish Council for Strategic Research (grant numbers 12-134923 to L.L.L. and 12-134922 to K.S.J.).

\section{Author contributions}

T.J.S. carried out most of the activity assays, assisted by T.Tr. and L.F.L.W. K.E.H.F. carried out most of the structural studies with J.C.P., T.Ta. and L.L.L. L.C. carried out
EPR spectroscopy. L.N. and B.H. carried out phylogenetic studies. M.T. and K.S. carried out target protein identification and production. P.D., L.L.L. and P.H.W. supervised the experimental work. T.J.S., K.E.H.F., K.S.J., P.H.W., L.L.L. and P.D. analysed the data and wrote the paper.

\section{Additional information}

Supplementary Information accompanies this paper at https://doi.org/10.1038/s41467017-01247-3.

Competing interests: M.T. and K.S. are employees of Novozymes, a producer of enzymes for industrial use. The remaining authors declare no conflict of interest.

Reprints and permission information is available online at http://npg.nature.com/ reprintsandpermissions/

Publisher's note: Springer Nature remains neutral with regard to jurisdictional claims in published maps and institutional affiliations.

(c) (i) Open Access This article is licensed under a Creative Commons Attribution 4.0 International License, which permits use, sharing, adaptation, distribution and reproduction in any medium or format, as long as you give appropriate credit to the original author(s) and the source, provide a link to the Creative Commons license, and indicate if changes were made. The images or other third party material in this article are included in the article's Creative Commons license, unless indicated otherwise in a credit line to the material. If material is not included in the article's Creative Commons license and your intended use is not permitted by statutory regulation or exceeds the permitted use, you will need to obtain permission directly from the copyright holder. To view a copy of this license, visit http://creativecommons.org/ licenses/by/4.0/.

(C) The Author(s) 2017 